\title{
A Lagrangian Cell-Centred Finite Volume Method for Metal Forming Simulation
}

\author{
P. Cardiff ${ }^{1 *}$, Ž. Tuković ${ }^{1,2}$, P. De Jaeger ${ }^{1,3}$, M. Clancy $^{1}$, and A. Ivanković ${ }^{1}$ \\ ${ }^{1}$ University College Dublin, Bekaert University Technology Centre, School of Mechanical and Materials Engineering, \\ Belfield, Ireland \\ ${ }^{2}$ University of Zagreb, Faculty of Mechanical Engineering and Naval Architecture, Croatia \\ ${ }^{3}$ NV Bekaert SA, Belgium
}

\begin{abstract}
SUMMARY
The current article presents a Lagrangian cell-centred finite volume solution methodology for simulation of metal forming processes. Details are given of the mathematical model in updated Lagrangian form, where a hyperelastoplastic $J_{2}$ constitutive relation has been employed. The cell-centred finite volume discretisation is described, where a modified discretised is proposed to alleviate erroneous hydrostatic pressure oscillations; an outline of the memory efficient segregated solution procedure is given. The accuracy and order of accuracy of the method is examined on a number of 2-D and 3-D elastoplastic benchmark test cases, where good agreement with available analytical and finite element solutions is achieved.
\end{abstract}

Received ...

KEY WORDS: Finite volume methods; Lagrangian; Plasticity; OpenFOAM

\section{INTRODUCTION}

1 For the past 40 years, there has been significant development of the finite element (FE) method for 2 metal forming problems. Traditionally, two distinct approaches have been adopted:

- Eulerian approach;

- Lagrangian approach.

5 As indicated schematically in Figure 1, the Eulerian approach follows a domain as material flows 6 through it; whereas, the Lagrangian approach follows material as it flows through a domain. In 7 practice, there are a number of hybrid methods displaying characteristics of both approaches, 8 such as the Arbitrary Lagrangian Eulerian (ALE) method. The first FE techniques to successfully 9 simulate metal forming processes adopted the Eulerian approach. These approaches assumed the 10 plastic strains to be considerably larger than the elastic strains; consequently, the idealisation

*Correspondence to: Philip Cardiff, University College Dublin, School of Mechanical and Materials Engineering, Belfield, Ireland. E-mail: philip.cardiff@ucd.ie 


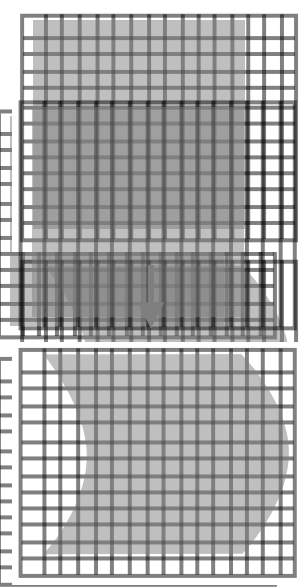

(a) Eulerian (fluid) formulation

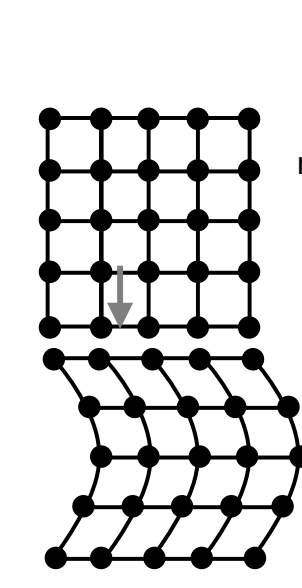

(b) Lagrangian (solid) formulation

Figure 1. Approaches to describe the initial and deformed configurations (adapted from [1] )

of rigid-plastic or rigid-viscoplastic material behaviour was acceptable. The main benefit of the Eulerian formulation is the ability to efficiently simulate extreme deformations with no concern for deteriorating mesh quality; however, drawbacks of the approach are the inability to predict the elastic response, memory effects, and residual stresses e.g. prediction of spring back is not possible. The flow formulation approach has been successfully applied to a large range of metal forming problems including extrusion, rolling, drawing, forging, analysis of slip and die design [2-6].

Owing to the success of the Eulerian approach and difficulties with extreme deformations, the successful application of the Lagrangian approach to metal forming problems developed later [3]. In the Lagrangian approach, the material is considered to be elastic-plastic or elastic-viscoplastic; as a result, the formulation can predict elastic phenomena, while also allowing capturing history dependent effects such as work hardening. The major drawbacks of the Lagrangian formulation, however, are severe mesh distortion, resulting in aborted simulation or decreased accuracy, and the requirement of large numbers of small time-steps to simulate quasi-static problems. To overcome mesh distortion, adaptive meshing and re-meshing algorithms are often employed, though, the procedures can be expensive and introduce appreciable errors through repeated solution re-mapping [5-9].

Although the FE method has been the dominant technique for forming simulations, there has been a number of developments of the FV method in this regard. Due to the prominence of the FV method in the field of Computational Fluid Dynamics, it is natural to expect the application of the FV method in Eulerian form to metal forming; a number of authors have applied FV-based flow formulations to simulate plastic deformation processes, e.g. see [10-18]. When examining the literature related to the application of Lagrangian FV methods to solid mechanics, a large range of practical problems have been successfully analysed [10-13, 19-56]; nonetheless, there has been relatively little research into Lagrangian FV approaches for finite strain elastoplastic analysis. Maneeratana and Ivanković [57-60] developed such a procedure, where a number of separate mathematical models were examined including those based on hypoelastoplastic relations with objective Jaumann 
and Truesdell stress rates; Bijelonja [61] later adopted a similar approach. Both Maneeratana [57] and Bijelonja [61] employed a spatially second-order cell-centred FV discretisation and implicit segregated solution methodology, based on the original work of Demirdžić and co-workers $[19,23]$. In recent years, there has been significant advances by Kluth and Després [52], Bonet and co-workers [53-55] on the development of explicit mixed formulation FV procedures for solid dynamics, where the linear momentum, deformation gradient tensor and total energy are the conservation variables. Such procedures bear their roots in Eulerian FV Godunov methods applied to compressible gas dynamics, and as such are well suited to shocks and discontinuities.

Building on the implicit Lagrangian FV approaches of Maneeratana [57] and Bijelonja [61], the current article extends the method to use a hyperelastoplastic-based approach, similar to those increasingly seen in FE implementations [8]. Combined with the development of frictional contact boundaries based on the penalty method, the current article is the first to present an implicit Lagrangian FV method with significant capabilities for metal forming simulation. The hyperelastoplastic theory $[8,62]$ is based on the $\boldsymbol{F}=\boldsymbol{F}^{e} \cdot \boldsymbol{F}^{p}$ multiplicative split of the deformation gradient into elastic and plastic components; the method has been applied in a number of forms of the FE method, e.g. see a recent isogeometric implementation in [63], as well as in explicit mixed FV formulations $[52,54,55]$. Unlike their popular hypoelastic-based counterparts, hyperelasticbased approaches are built on sound thermodynamical principles, where there is no net work done in a closed cycle of elastic deformation [64].

The article is constructed as follows: Section 2 outlines the updated Lagrangian mathematical model, derived from the governing momentum equation and hyperelastoplastic constitutive relation. The discretisation of the mathematical model using a spatially second-order accurate cell-centred FV method is presented in Section 3, along with details of the implicit iterative solution procedure and frictional contact boundary conditions. In Section 4, the new method is evaluated on six separate benchmark test cases, where predictions are compared to analytical solutions and FE benchmarks.

\section{MATHEMATICAL MODEL}

\subsection{Governing Equations}

Considering an arbitrary body of volume $\Omega$ bounded by surface $\Gamma$ with outward pointing unit normal $\boldsymbol{n}$, the conservation of linear momentum is given in strong integral form as:

$$
\frac{\mathrm{d}}{\mathrm{d} t} \int_{\Omega} \rho \boldsymbol{v} \mathrm{d} \Omega=\oint_{\Gamma} \boldsymbol{n} \cdot \boldsymbol{\sigma} \mathrm{d} \Gamma+\int_{\Omega} \rho \boldsymbol{b} \mathrm{d} \Omega
$$

where $\rho$ is the density, $\boldsymbol{v}$ is the velocity vector, $\boldsymbol{\sigma}$ is the Cauchy stress tensor, and $\boldsymbol{b}$ is a body force per unit mass.

\subsection{Constitutive Relation}

In the current study, a rate-independent isotropic hyperelastoplastic constitutive relation between stress and strain is employed, as outlined in Simo and Hughes [8], where the deformation gradient $\boldsymbol{F}=\boldsymbol{F}^{e} \cdot \boldsymbol{F}^{p}$ is multiplicatively decomposed into elastic and plastic components. The Kirchhoff 
stress $\tau$, related to the Cauchy stress $\tau=J \sigma$, is given in terms of an uncoupled volumetricdeviatoric relation:

$$
\boldsymbol{\tau}=\frac{K}{2}\left(J^{2}-1\right) \mathbf{I}+\mu \operatorname{dev}\left[\overline{\boldsymbol{b}}^{e}\right]
$$

where the first term on the right-hand side is the volumetric component and the second term on the right-hand side is the deviatoric component. Assuming incompressible (isochoric) plastic deformation, the Jacobian of the elastic deformation gradient $J^{e}$ and the Jacobian of the deformation gradient $J$ coincide i.e. $J^{e}=J=\operatorname{det}[\boldsymbol{F}]$. The bulk modulus is $K$; the shear modulus is $\mu$; $\mathbf{I}$ is the second-order identity tensor; the elastic left Cauchy-Green deformation tensor is $\boldsymbol{b}^{e}$, where the volume preserving component $\bar{b}^{e}$ is given as:

$$
\overline{\boldsymbol{b}}^{e}=J^{-\frac{2}{3}} \boldsymbol{b}^{e}=J^{-\frac{2}{3}} \boldsymbol{F}^{e} \cdot \boldsymbol{F}^{e T}
$$

In the limit of small strains, Equation 2 reduces to the standard form of Hooke's law.

The classical Mises-Huber $J_{2}$ yield condition, formulated in terms of the Kirchhoff stress tensor, is employed:

$$
f\left(\tau, \epsilon^{p e q}\right)=\|\operatorname{dev}[\tau]\|-\sqrt{\frac{2}{3}}\left[\sigma_{Y}\left(\epsilon^{p e q}\right)\right] \leq 0
$$

where the yield stress $\sigma_{Y}$ is a function of the hardening variable, equivalent plastic strain $\epsilon^{p e q}$. In the current implementation, the isotropic hardening curve for the yield stress is described in a piecewise linear manner; as such, the yield function can in general be a nonlinear function of the hardening parameter. The associative flow rule takes the form:

$$
\begin{aligned}
\frac{\delta}{\delta t}\left(\overline{\boldsymbol{F}}^{-1} \cdot \overline{\boldsymbol{b}}^{e} \cdot \overline{\boldsymbol{F}}^{-T}\right) & =-\frac{2}{3} \gamma \operatorname{tr}\left[\boldsymbol{b}^{e}\right] \boldsymbol{F}^{-1} \cdot \boldsymbol{N} \cdot \boldsymbol{F}^{-T} \\
\boldsymbol{N} & =\frac{\boldsymbol{s}}{\|\boldsymbol{s}\|}
\end{aligned}
$$

where $\boldsymbol{N}$ is the return direction and $s$ is the deviatoric component of the stress tensor $s=\operatorname{dev}[\boldsymbol{\tau}]$. The evolution of the hardening variable is governed by the rate equation:

$$
\dot{\epsilon}^{p e q}=\sqrt{\frac{2}{3}} \gamma
$$

The consistency parameter $\gamma$ is subject to the standard Kuhn-Tucker loading/unloading conditions:

$$
\gamma \geq 0, \quad f\left(\tau, \epsilon^{p e q}\right) \leq 0, \quad \gamma f\left(\tau, \epsilon^{p e q}\right)=0
$$

which along with the consistency condition:

$$
\gamma \dot{f}\left(\tau, \epsilon^{p e q}\right)=0
$$

complete the formulation of the model. The current constitutive relation can be extended to kinematic hardening and thermoplasticity following the approaches of Simo and Hughes [8] and Simo and Miehe [65]. 


\subsection{Updated Lagrangian Formulation}

By employing Nanson's relation [57, 62], relating the deformed $\Gamma$ and initial $\boldsymbol{\Gamma}_{o}$ area vectors $\boldsymbol{\Gamma}=J \boldsymbol{F}^{-T} \cdot \boldsymbol{\Gamma}_{o}$, the conservation of linear momentum (Eq. 1) may be reformulated in terms of the initial undeformed configuration, indicated by subscript $o$ :

$$
\int_{\Omega_{o}} \frac{\partial}{\partial t}\left(\rho_{o} \boldsymbol{v}\right) \mathrm{d} \Omega_{o}=\oint_{\Gamma_{o}}\left(J \boldsymbol{F}^{-T} \cdot \boldsymbol{n}_{o}\right) \cdot \boldsymbol{\sigma} \mathrm{d} \Gamma_{o}
$$

or equivalently in terms of the updated configuration, indicated by subscript $u$ :

$$
\begin{aligned}
\int_{\Omega_{u}} \frac{\partial}{\partial t}\left(\rho_{u} \boldsymbol{v}\right) \mathrm{d} \Omega_{u} & =\oint_{\Gamma_{u}}\left(j \boldsymbol{f}^{-T} \cdot \boldsymbol{n}_{u}\right) \cdot \boldsymbol{\sigma} \mathrm{d} \Gamma_{u} \\
& =\oint_{\Gamma_{u}}\left(\frac{j}{J} \boldsymbol{f}^{-T} \cdot \boldsymbol{n}_{u}\right) \cdot \boldsymbol{\tau} \mathrm{d} \Gamma_{u}
\end{aligned}
$$

where the relative deformation gradient $f$ relates the current deformation state and the previous deformation state, referred to as the updated state. The Jacobian of the relative deformation gradient, termed the relative Jacobian, is $j=\operatorname{det}[\boldsymbol{f}]$. The relative deformation gradient is given in terms of the displacement increment as $\boldsymbol{f}=\mathbf{I}+\boldsymbol{\nabla}(\Delta \boldsymbol{u})^{T}$. Equation 10 may be written in terms of the unknown displacement increments, which are considered the primary unknown variables.

In the current FV approach, both total Lagrangian (TL) and updated Lagrangian (UL) forms can be implemented; the updated Lagrangian has been selected as it gave superior robustness and convergence in initial numerical trials. In theory the TL and UL forms can be implemented in an equivalently robust manner; however, in the current procedure, the use of a segregated algorithm necessitates the implementation of iterative traction boundary conditions; the TL approaches iterates over traction terms dependent on the total deformation gradient $\boldsymbol{F}$, whereas the UL implementation iterates over traction terms dependent on the relative deformation gradient $f$, resulting in the UL implementation providing a more stable implicit solution procedure.

\section{NUMERICAL METHOD}

In this section, the mathematical model presented in the preceding section is discretised using the cell-centred FV method, synonymous with the collocated FV method, providing a discrete approximation of the previously presented exact integrals. Unlike standard FE methods where the governing equation in strong form is cast into its equivalent weak form, the current FV method directly discretises the strong integral form of the governing equation. The discretisation procedure is separated into two distinct parts: discretisation of the solution domain, and discretisation of the governing equations.

\subsection{Solution Domain Discretisation}

Discretisation of the solution domain comprises the discretisation of time and the discretisation of space. The total specified simulation time is divided into a finite number of time increments, $\Delta \mathrm{t}$, and the discretised governing equations are solved in a time-marching manner. The solution domain 
space is divided into a finite number of contiguous convex polyhedral cells bounded by polygonal faces, that do not overlap and fill the space completely. A typical control volume (or cell or control mass), shown in Figure 2, has a computational node $P$ located at the cell centroid, with cell volume $\Omega_{P}, N$ is the centroid of a neighbouring control volume, face $f$ has area vector $\boldsymbol{\Gamma}_{f}$, vector $\boldsymbol{d}_{f}$ joins $P$ to $N$ and $\boldsymbol{r}$ is the positional vector of $P$. No distinction is made between different cell volume shapes, as all general polyhedra (e.g. tetrahedra, hexahedra, triangular prism, dodecahedra, etc.) are discretised in the same general fashion; this is in contrast to standard FE methods where shape functions are specific to the shape of the element.

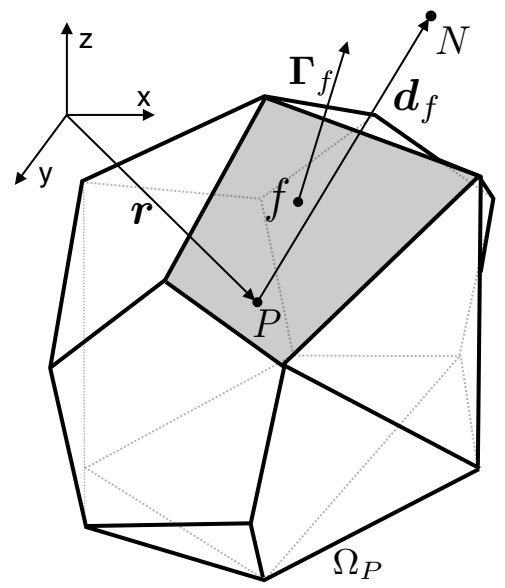

Figure 2. General convex polyhedral control volume (adapted from $[66,67]$ )

\subsection{Equation Discretisation}

To allow use of a segregated/staggered solution procedure, the surface force (diffusion term) is partitioned into implicit and explicit components:

$$
\begin{aligned}
\int_{\Omega_{u}} \frac{\partial}{\partial t}\left(\rho_{u} \frac{\partial\left(\boldsymbol{u}^{[m-1]}+\Delta \boldsymbol{u}\right)}{\partial t}\right) \mathrm{d} \Omega_{u}= & \oint_{\Gamma_{u}}\left(\frac{4}{3} \mu+K\right) \boldsymbol{n}_{u} \cdot \nabla(\Delta \boldsymbol{u}) \mathrm{d} \Gamma_{u} \\
& +\oint_{\Gamma_{u}}\left(j \boldsymbol{f}^{-T} \cdot \boldsymbol{n}_{u}\right) \cdot \boldsymbol{\sigma} \mathrm{d} \Gamma_{u}-\oint_{\Gamma_{u}}\left(\frac{4}{3} \mu+K\right) \boldsymbol{n}_{u} \cdot \nabla(\Delta \boldsymbol{u}) \mathrm{d} \Gamma_{u}
\end{aligned}
$$

where the first term on the right-hand side is treated implicitly and the second and third terms on the right-hand side are treated explicitly; in this context, by implicit it is meant that the term contributes coefficients to the matrix of the resulting linear system of equations, whereas by explicit it is implied that the term is calculated using the latest known values and solely contributes to the source vector of the resulting linear system; the displacement increment $\Delta \boldsymbol{u}=\boldsymbol{u}^{[m]}-\boldsymbol{u}^{[m-1]}$ is the primary unknown; superscript $[m]$ indicates values at the current time increment, superscript $[m-1]$ indicates values at the previous/old time increment. As outlined later, outer Picard/FixedPoint iterations provide the necessary implicit coupling at each time-step. This approach echoes 
the methods of Demirdžić and Martinović [23] and Jasak and Weller [29], where the displacement inter-component coupled terms and nonlinear terms are treated in a deferred correction manner.

For each cell $P$, the temporal term is discretised using a first-order accurate in time fully implicit backward Euler finite differencing scheme:

$$
\int_{\Omega_{u}} \frac{\partial}{\partial t}\left(\rho_{u} \frac{\partial(\Delta \boldsymbol{u})}{\partial t}\right) \mathrm{d} \Omega_{u} \approx \frac{1}{\Delta t^{[m]}}\left[\left(\rho_{u} \Omega\right)_{P}^{[m]}\left(\frac{\Delta \boldsymbol{u}_{P}^{[m]}-\Delta \boldsymbol{u}_{P}^{[m-1]}}{\Delta t^{[m]}}\right)-\left(\rho_{u} \Omega\right)_{P}^{[m-1]}\left(\frac{\Delta \boldsymbol{u}_{P}^{[m-1]}-\Delta \boldsymbol{u}_{P}^{[m-2]}}{\Delta t^{[m-1]}}\right)\right]
$$

The component of the temporal term containing $\boldsymbol{u}_{\text {old }}$ is discretised in a similar fashion to Equation 12 but the term is calculated in an entirely explicit manner i.e. the term contributes solely to the source vector of the resulting linear system of equations. It is straight-forward to discretise the temporal term using a second-order accurate scheme, as shown for the current FV method in Jasak and Weller [29]; however, the temporal order of accuracy of the current elastoplastic method is limited by the first-order backward Euler integration of the plastic deformation rate within the constitutive law; the integration error in the plastic strain could be controlled using an adaptive time-step procedure, as outlined for example by Lee and Bathe [68].

The implicit diffusion term (first term on the right-hand side of Equation 11) is discretised using central differencing with over-relaxed non-orthogonal correction [23, 29, 40]:

$$
\begin{aligned}
\oint_{\Gamma_{u}}\left(\frac{4}{3} \mu+K\right) \boldsymbol{n}_{u} \cdot \boldsymbol{\nabla}(\Delta \boldsymbol{u}) \mathrm{d} \Gamma_{u} \approx & \sum_{F}\left(\frac{4}{3} \mu_{f}+K_{f}\right)\left|\boldsymbol{\Delta}_{u f}\right|\left(\frac{\boldsymbol{u}_{N}-\boldsymbol{u}_{P}}{\left|\boldsymbol{d}_{f}\right|}\right)\left|\boldsymbol{\Gamma}_{u f}\right| \\
& +\sum_{F}\left(\frac{4}{3} \mu_{f}+K_{f}\right) \boldsymbol{k}_{u f} \cdot[\boldsymbol{\nabla}(\Delta \boldsymbol{u})]_{f}\left|\boldsymbol{\Gamma}_{u f}\right|
\end{aligned}
$$

where $F$ is the number of internal faces in cell $P, \boldsymbol{\Delta}_{u f}=\frac{\boldsymbol{d}_{u f}}{\boldsymbol{d}_{u f} \cdot \boldsymbol{n}_{u f}}, \boldsymbol{k}_{u f}=\boldsymbol{n}_{u f}-\boldsymbol{\Delta}_{u f}$, and $\boldsymbol{n}_{u f}$ is the unit normal of the face. The first term on the right-hand side of the equation is treated implicitly, whereas the second term on the right-hand side, representing a correction for the face non-orthogonality, is treated explicitly; further details are given in [23, 29, 40].

The explicit surface diffusion source terms (second and third terms on the right-hand side of Equation 11) are discretised by assuming a linear variation of the term across the face:

$$
\begin{aligned}
\oint_{\Gamma_{u}}\left(j \boldsymbol{f}^{-T} \cdot \boldsymbol{n}_{u}\right) \cdot \boldsymbol{\sigma} \mathrm{d} \Gamma_{u} & =\sum_{F} \int_{\Gamma_{u f}}\left(j \boldsymbol{f}^{-T} \cdot \boldsymbol{n}_{u}\right) \cdot \boldsymbol{\sigma} \mathrm{d} \Gamma_{u f} \\
& \approx \sum_{F} \boldsymbol{\Gamma}_{u f} \cdot\left(j \boldsymbol{\sigma} \cdot \boldsymbol{f}^{-T}\right)_{f}
\end{aligned}
$$

and similarly:

$$
\begin{aligned}
\oint_{\Gamma_{u}}\left(\frac{4}{3} \mu+K\right) \boldsymbol{n}_{u} \cdot \boldsymbol{\nabla}(\Delta \boldsymbol{u}) \mathrm{d} \Gamma_{u} & =\sum_{F} \int_{\Gamma_{u f}}\left(\frac{4}{3} \mu+K\right) \boldsymbol{n}_{u} \cdot \boldsymbol{\nabla}(\Delta \boldsymbol{u}) \mathrm{d} \Gamma_{u f} \\
& \approx \sum_{F}\left(\frac{4}{3} \mu+K\right)_{f} \boldsymbol{\Gamma}_{u f} \cdot[\nabla(\Delta \boldsymbol{u})]_{f}
\end{aligned}
$$


Terms at a face, indicated by subscript $f$, are linearly interpolated from adjacent cell-centre values; cell-centre gradients are calculated using a least squares method (extendedLeast Squares scheme in foam-extend-3.2). It should be noted that the first term on the right-hand side of Equation 11, treated implicitly, and third term on the right-hand side of Equation 11, treated explicitly, do not exactly cancel out as they employ different computational stencils; the difference between the two terms results in additional numerical diffusion, which tends to eliminate erroneous oscillations in the solution variable field, as initially proposed by Rhie and Chow [69].

Further details of the discretisation of the temporal, implicit and explicit terms can be found in $[29,40]$, for example. The resulting discretisation is spatially second-order accurate, that is, the discretisation error reduces at a second-order rate as the cell size is reduced. In contrast to conventional FE methods, the current cell-centred FV discretisation is strongly conservative, both locally and globally.

Although not the focus of the current study, it is worth briefly discussing the extension of the current formulation to account for discontinuous material property fields, i.e. where there exists internal bi-material interfaces with step changes in material properties (e.g. a jump in shear modulus and/or Poisson's ratio): if the current discretisation, as is, was applied to such a case, erroneous peaks in the strain/stress in the cells immediately adjacent to the interface would be expected. One approach to overcome this issue for the current cell-centred FV method was proposed by Tuković et al. [37], where a modification of the traction discretisation at the bi-material interface was successfully employed. Applying a similar modification here would allow for discontinuous material property fields to be handled in the current discretisation.

As regards the physical shocks (discontinuities in the solution field), the current discretisation employs central differencing and, as such, would suffer from inaccuracies present in other central differencing schemes when applied to shocks. Extending the current discretisation to employ upwind-style methods would be one potential solution.

Hydrostatic Pressure Contribution Displacement-based procedures, like the current FV method, have been found effective for a wide variety of problems in the realm of FE; there are, however, situations when such displacement-based approaches tend to be less effective, namely, in the analysis of incompressible or quasi-incompressible materials [62]; as experienced, for example, in large plastic deformation of metals. In such situations, hybrid/mixed approaches are favoured, where displacement and pressure are the typical unknowns. Given the close relationship of the FV method with the FE method, it is unsurprising that the current FV procedure would suffer from similar issues with pressure oscillations. The reason for the appearance of such numerical oscillations can be explained by the volumetric strains being very small in comparison to the deviatoric strains; as the strains are determined from the derivatives of displacements, which are not as accurately predicted as the displacements, errors in the volumetric strains can appear as appreciable errors in the hydrostatic stresses; for example see the discussion by Bathe [62].

Of course extending the current approach to employ a hybrid approach would be one approach to mitigate this problem (and this will be considered in future developments); however, in the current article a modified discretisation of the hydrostatic pressure term is examined in an attempt to minimise these pressure oscillations. 
In the current updated Lagrangian formulation the hydrostatic pressure is a function of the relative Jacobian $j$; in the standard discretisation approach described above, the relative Jacobian $j$ is calculated at each cell-centre as:

$$
\begin{aligned}
j & =\operatorname{det}[\boldsymbol{f}] \\
& =\operatorname{det}\left[\mathbf{I}+\boldsymbol{\nabla}(\Delta \boldsymbol{u})^{T}\right]
\end{aligned}
$$

where the cell-centre gradient of the displacement increment $\nabla(\Delta \boldsymbol{u})$ is calculated using a linear least squares method. In the proposed modified approach, the relative Jacobian $j$ is calculated as:

$$
\begin{aligned}
j & =\operatorname{det}[\boldsymbol{f}] \\
& =\operatorname{det}\left[\mathbf{I}+\widehat{\nabla(\Delta \boldsymbol{u})}^{T}\right]
\end{aligned}
$$

where the modified gradient of displacement increment term $\widehat{\nabla(\Delta \boldsymbol{u})}$, at the cell-centres, is calculated as:

$$
\widehat{\boldsymbol{\nabla}(\Delta \boldsymbol{u})}=\left(\sum_{F} \frac{1}{\left|\boldsymbol{\Gamma}_{f}\right|} \boldsymbol{\Gamma}_{f} \boldsymbol{\Gamma}_{f}\right)^{-1} \cdot\left(\sum_{F} \boldsymbol{\Gamma}_{f}[\boldsymbol{n} \cdot \boldsymbol{\nabla}(\Delta \boldsymbol{u})]_{f}\right)
$$

The term $[\boldsymbol{n} \cdot \boldsymbol{\nabla}(\Delta \boldsymbol{u})]_{f}$ represents the normal gradient of displacement increment calculated directly at the control volume face; this term is approximated using central differencing, as in Equation 13. The procedure in Eq. 18, developed by Weller et al. [28], is termed a reconstruction operation in the OpenFOAM software (fvc: : reconstruct in the OpenFOAM code syntax), where in CFD applications it can be employed to approximate the cell-centred velocity field from the face-centre flux field (normal component of velocity at the face); details of the derivation of this approximation are given in Appendix A. In the current application, the procedure is employed as a form of spatial smoothing, where the gradient is calculated at the faces of the cell and reconstructed to the cellcentre; the term is then linearly interpolated back to the cell faces to calculate the hydrostatic pressure contributions at the cell faces; as will be shown in the test cases section, this modification has been found to reduce the propensity for pressure oscillations to occur.

\subsection{Initial and Boundary conditions}

All dependent variables have to be specified at the initial time $t_{0}$. On the faces coinciding with the boundary of the solution domain, boundary conditions have to be applied. In the case of a Dirichlet boundary condition, the expressions for diffusion fluxes and sources remain valid, except for employing the user specified boundary value in place of the neighbouring cell-centre value. On the boundaries where Neumann boundary conditions are specified, the boundary fluxes are added to the source term, while the variable values at the boundary are extrapolated from the internal domain using the specified boundary gradient. Further details of the boundary condition implementation can be found in, for example, [40].

Frictional Contact Boundary Conditions In the current method, contact boundary conditions are implemented in the form of Neumann conditions, where the contact constraints are enforced 
using a penalty method. A frictionless implementation presented previously [36] has been extended to include Coulomb friction, where the tangential traction (friction) is calculated as:

$$
\boldsymbol{T}_{\text {fric }}=\left\{\begin{array}{ccc}
-k_{\text {fric }} \gamma & \text { for } & \boldsymbol{T}_{\text {fric }}<\mu_{\text {fric }} \sigma_{n} \\
-\mu_{\text {fric }}\left|\sigma_{n}\right| \frac{\gamma}{|\gamma|} & \text { for } & \boldsymbol{T}_{\text {fric }} \geq \mu_{\text {fric }} \sigma_{n}
\end{array}\right.
$$

where the Coulomb coefficient of friction is $\mu_{\text {fric }}$, the normal contact stress $\sigma_{n}=\boldsymbol{n} \cdot(\boldsymbol{n} \cdot \boldsymbol{\sigma})$, the slip $\gamma$ is calculated as the difference in displacement increment between one contacting surface and the corresponding point on the other contacting surface in the surface tangential direction i.e. the slip has no component in the surface normal direction $\boldsymbol{n} \cdot \boldsymbol{\gamma}=0$; the friction penalty factor is set as:

$$
k_{\text {fric }}=\frac{f_{\text {scale }} \mu A}{V}
$$

where $\mu$ is the average shear modulus of the faces on the contact boundary (not to be confused with the Coulomb coefficient of friction), $A$ is the average area of the faces on the contact boundary and $\mathrm{V}$ is the average volume of cells at the contact boundary. The scaling factor, $f_{\text {scale }}$, is typically set to 1.0 but can be increased if there is noticeable elastic slip i.e. slip before the critical slip traction is reached. Although the Coulomb friction relation has been selected here, it would be straightforward to include other relations, such as Tresca, Coulomb-Orowan, Shaw, or laws dependent on temperature/pressure and other solution variables.

\subsection{Solution Procedure}

The final discretised form of the linear momentum equation for each control volume $P$ can be arranged in the form of $M$ linear algebraic equations, e.g. see [29, 40]:

$$
a_{P} \Delta \boldsymbol{u}_{P}+\sum_{F} a_{N} \Delta \boldsymbol{u}_{N}=\boldsymbol{b}_{P}
$$

where $F$ is the number of internal faces of control volume $P, a_{P}$ is the central coefficient, $a_{N}$ are the neighbour coefficients representing interactions with neighbour cell-centred unknowns, and $\boldsymbol{b}_{P}$ is the source vector contribution.

The algebraic linear equations described above are assembled for all control volumes forming a system of linear equations:

$$
[\boldsymbol{A}][\boldsymbol{\phi}]=[\boldsymbol{b}]
$$

where $[\boldsymbol{A}]$ is a sparse $M \times M$ matrix with weak diagonal dominace, with $M$ being the total number of control volumes. The coefficients $a_{P}$ are on the diagonal of matrix $[\boldsymbol{A}]$ and coefficients $a_{N}$ form the matrix off-diagonal. The solution vector $[\phi]$ contains the unknown cell-centre displacement increments $\Delta \boldsymbol{u}$, and $[\boldsymbol{b}]$ is the source vector containing the explicit discretised terms and boundary condition contributions

The linear system of equations are solved in a segregated manner, with each component of the displacement increment field solved for separately. Outer Picard/Fixed-Point iterations are performed to account for the inter-equation coupling and the linearised nonlinear terms. The inner linear sparse system is iteratively solved, typically using the incomplete Cholesky pre-conditioned conjugate gradient (ICCG) method [70]. The inner system need not be solved to a tight tolerance 
as coefficients and source terms are approximated from the previous increment; a reduction in the residuals of one order of magnitude is typically sufficient. The outer iterations are performed until the predefined tolerance, typically $1 \times 10^{-6}$, has been achieved. The current method has been implemented in open-source software OpenFOAM [28] (foam-extend-3.2), exploiting the developed object oriented parallelised $\mathrm{FV}$ procedures.

It should be noted that standard implicit nonlinear FE methods typically use a full or modified Newton-Raphson loop to account for the nonlinearities, necessitating the need for a consistent tangent matrix for optimal convergence [62]; in contrast, the current method employs Picard/FixedPoint iterations to account for the nonlinearities and hence does not require a consistent tangent matrix. The Newton method can achieve quadratic convergence compared with the linear convergence rate of the Picard iterations, and consequently a larger number of total iterations would be expected in the current implementation; however, through the use of efficient iterative linear solvers, each outer iteration is much less expensive, resulting in a competitive method.

The overall solution procedure is summarised in Algorithm 1. The procedure to update the kinematics and stress is given in Algorithm 2, echoing the procedure presented in Simo and Hughes [8], where subscript $m-1$ implies quantities from the previous time-step and subscript $m$ implies quantities from the current time-step; at line 12 the plastic multiplier $\Delta \gamma$ is iteratively calculated by solving Equation 23 using a Newton loop, where in general the yield stress $\sigma_{Y}$ is a function of the plastic multiplier. A point to note is that the hydrostatic pressure term calculated at line 20 is computed directly from the displacement field; in contrast, hybrid pressure-displacement approaches would solved an additional governing equation to obtain this pressure.

One disadvantage of the cell-centred FV discretisation relative to conventional FE methods is that the calculated displacements reside at the cell-centres, whereas displacements at the vertices are needed to move the mesh; consequently, the cell-centred displacements must be interpolated to the vertices before moving the mesh at the end of the time-step; in the current approach, a linear least squares interpolation is employed [40, 48, 71]: at each mesh vertex, a linear least squares plane is fit through the displacement values at the immediately adjacent cell-centres; for vertices on the domain boundary, boundary face-centre values are also included in the fitting.

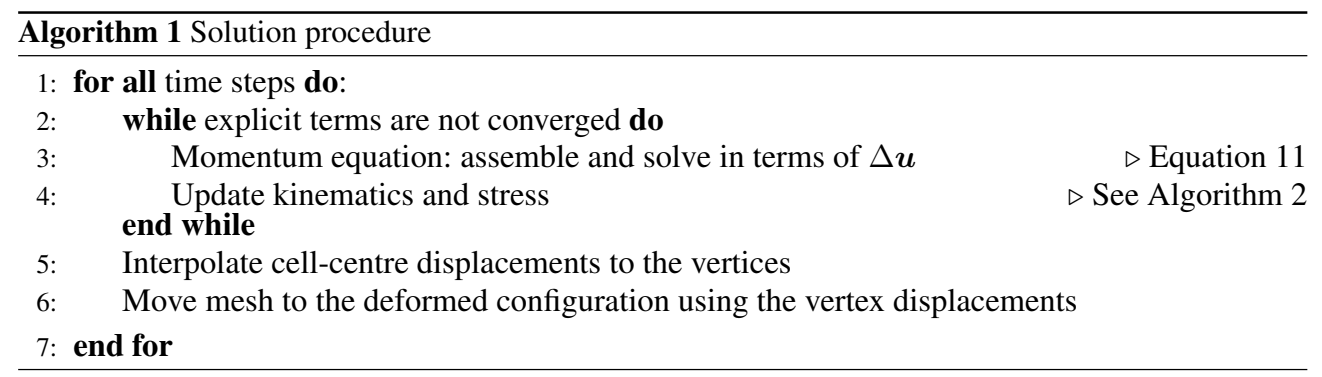

$$
f(\Delta \gamma)=\left\|s_{m}^{\text {trial }}\right\|-2 \bar{\mu} \Delta \gamma-\sqrt{\frac{2}{3}} \sigma_{Y}\left(\epsilon_{m-1}^{p e q}+\sqrt{\frac{2}{3}} \Delta \gamma\right)=0
$$




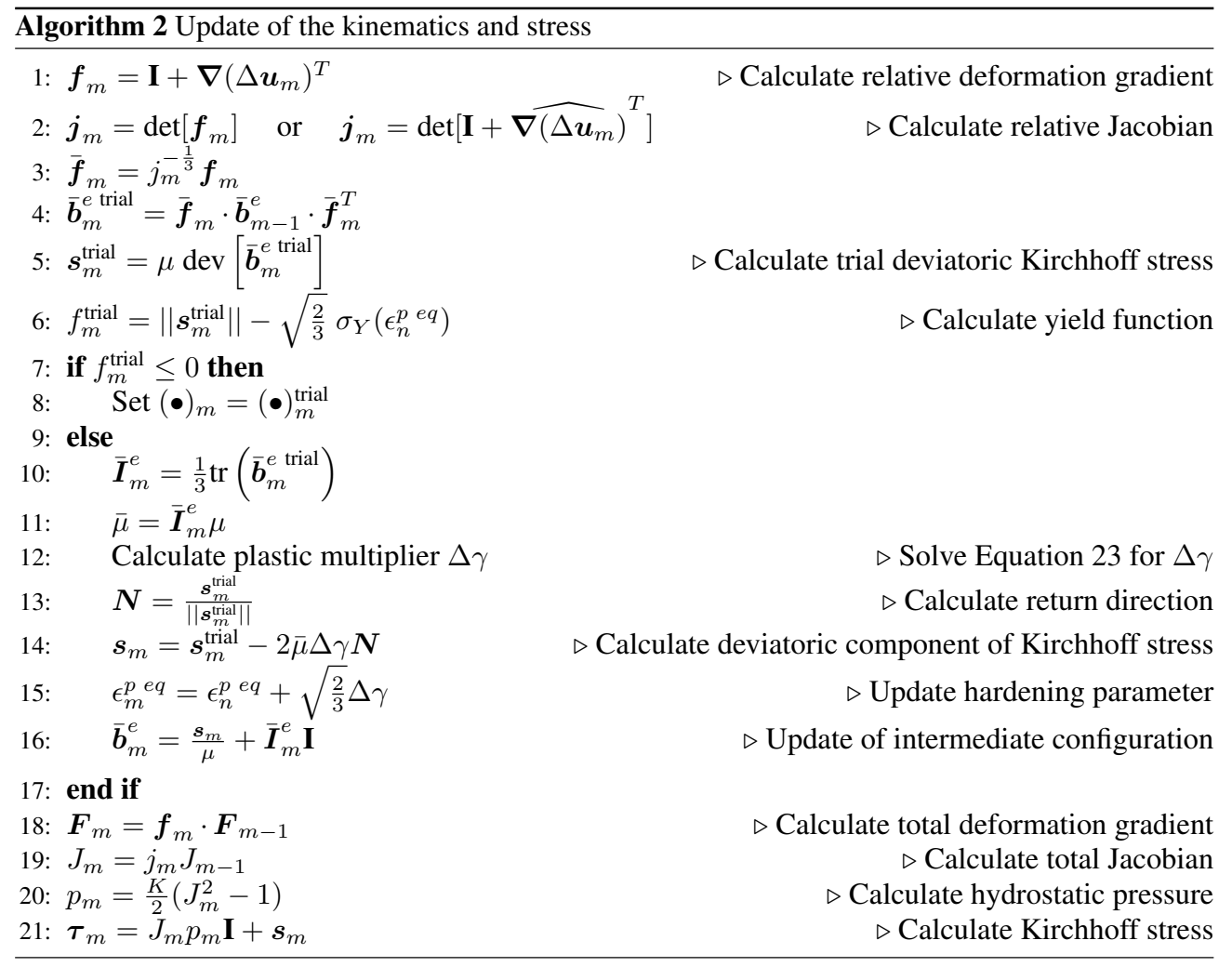

\section{NUMERICAL TEST CASES}

In this section, the capabilities of the developed FV methodology are examined on six elastoplastic benchmark cases:

1. Expansion of a thick-wall cylinder - 1-D axisymmetric;

2. Upsetting of a billet - 2-D axisymmetric - involving considerable mesh deformation and folding;

3. Crushing of a long cylindrical pipe - 2-D plane strain;

4. Necking of a cylindrical bar-2-D axisymmetric;

5. Flat rolling of a cylindrical wire - evaluating the capabilities of the method for a nontrivial 3-D problem;

6. Cylinder impacting a rigid wall - highlighting the capabilities of the method for impact scenarios.

Temperature and gravity effects are neglected in all cases.

\subsection{Expansion of Thick-Walled Cylinder}

This 1-D axisymmetric test case has been considered by a number of authors, such as Simo and Hughes [8] and Taylor [72]: a thick-walled cylinder, with an inner radius $r_{i}$ of $10 \mathrm{~mm}$ and an outer radius $r_{o}$ of $20 \mathrm{~mm}$, subjected to internal expansion, see Figure 3(a). The mechanical properties, 
shown in Table I, are chosen to approximate rigid plastic behaviour allowing comparison with the exact analytical solution. Transient effects are neglected. Five separate systematically refined uniform quadrilateral axisymmetric meshes are considered: 5, 10, 20, 40 and 80 cells; the middle density mesh is shown in Figure 3(b). The inner radius is displaced by $75 \mathrm{~mm}$ in the radial direction, driving the inner cylinder radius to a final value of $85 \mathrm{~mm}$. A zero traction condition is applied to the outer cylinder boundary.

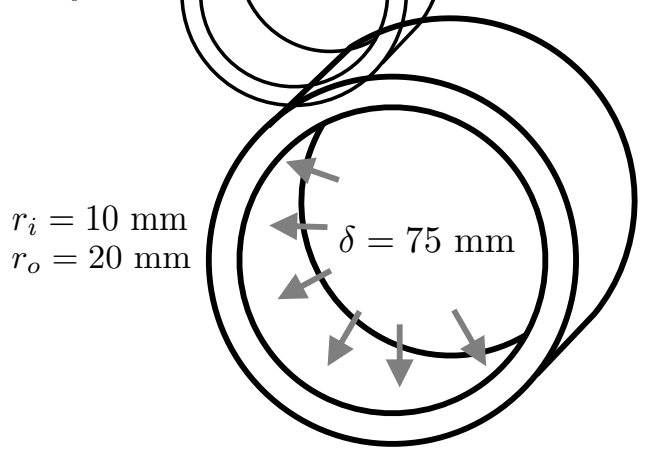

(a) Geometry

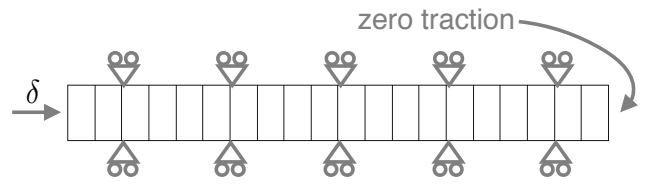

(b) 1-D axisymmetric mesh

Figure 3. Expansion of thick-walled cylinder: problem geometry, loading and mesh

\begin{tabular}{lll}
\hline Shear modulus & $\mu$ & $3.8 \mathrm{GPa}$ \\
Bulk modulus & $\kappa$ & $40 \mathrm{GPa}$ \\
Yield stress & $\sigma_{Y}$ & $0.5 \mathrm{MPa}$ \\
Perfect plasticity & hardening parameter & 0.0
\end{tabular}

Table I. Expansion of thick-walled cylinder: mechanical properties

The models have been solved in double precision using $1 \mathrm{CPU}$ core $(2.4 \mathrm{GHz}$ Intel Ivy Bridge cores), over 15000 quasi-static time increments. The wall-clock time required ranged from $1 \mathrm{~min}$ for the coarsest mesh to $5 \mathrm{~min}$ for the finest mesh.

Assuming rigid plasticity, the radial (Cauchy) stress, $\sigma_{r r}$, at the inner surface is given analytically as [73, 74]:

$$
\sigma_{r r}=\left(\frac{\sigma_{Y}}{\sqrt{3}}\right) \ln \left(\frac{\left(\frac{r_{0}}{a_{0}}\right)^{2}+\left(\frac{a}{a_{0}}\right)^{2}-1}{\left(\frac{b_{0}}{a_{0}}\right)^{2}+\left(\frac{a}{a_{0}}\right)^{2}-1}\right)
$$

where $a_{0}$ is the initial inner radius; $b_{0}$ is the initial outer radius; $r_{0}$ is radius in the initial configuration of the material point at which the stresses are being calculated; and $a$ is the current value of the 


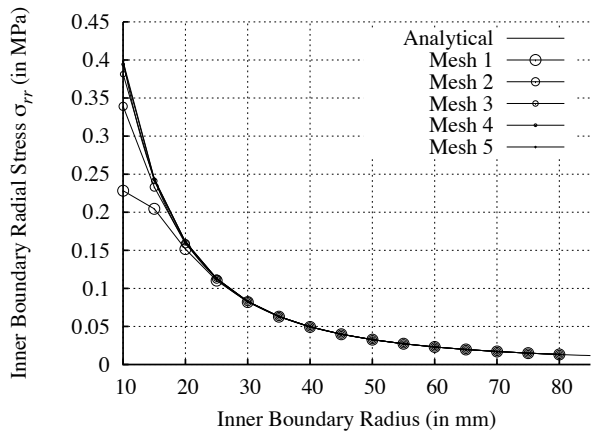

(a) Inner boundary stress $\sigma_{r r}$ vs. current inner radius

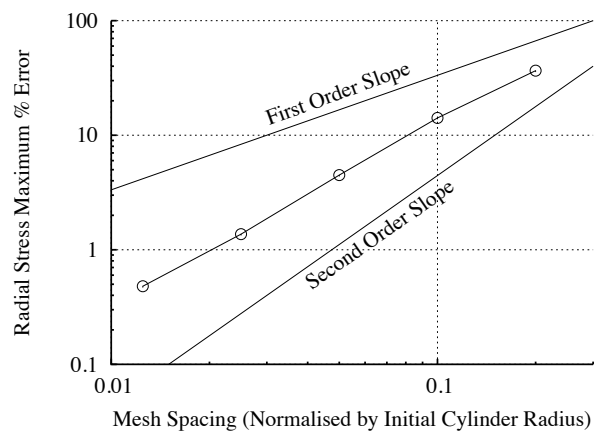

(b) Maximum error in $\sigma_{r r}$ at time $0.001 \mathrm{~s}$ as the mesh spacing is successively reduced

Figure 4. Expansion of thick-walled cylinder: radial stress $\sigma_{r r}$ and maximum error

inside radius. The hoop stresses and axial stresses are offset from the radial stress by a constant consequently only the radial stress is examined.

A plot of the radial (Cauchy) stress $\sigma_{r r}$ at the inner boundary versus the current inner radius is given in Figure 4(a), where for the current case the modified form of the relative Jacobian calculation is employed. Using the maximum difference between the predicted stress and the analytical solution as a metric, the accuracy and order of accuracy of the current method may be assessed. Figure 4(b) shows the maximum error in radial stress to reduce at approximately a second-order rate, in agreement with the second-order discretisation. The largest error can be seen to occur when the inner boundary deformed radius is near to its initial radius. As the inner boundary radius increases past approximately $30 \mathrm{~mm}$, it can be seen that all meshes are in excellent agreement with the exact solution.

\subsection{Upsetting a Billet}

The upsetting of a cylindrical billet between parallel rough dies has been used a number of times to test the validity of numerical methods for analysis of metal forming problems, e.g. [72, 74, 75] The billet, with initial diameter, $\phi=20 \mathrm{~mm}$, and initial height, $h=30 \mathrm{~mm}$, is upset by $60 \%$, corresponding to the a die displacement $\delta=18 \mathrm{~mm}$. The problem geometry and loading are illustrated in Figure 5(a). The material properties are given in Table II. Transient effects are neglected. The contact between the die and billet is rough and is approximated here using a penalty method contact procedure with a Coulomb friction coefficient of 0.5; Taylor [72] showed that such a high coefficient of friction sufficiently captures the rough surface approximation. The problem is represented as 2-D axisymmetric where only the top half of the geometry is modelled. Five separate successively refined meshes are examined, consisting of 36, 144, 576, 2304 and 9216 quadrilateral cells; the coarsest mesh is shown in Figure 5(b).

The models have been solved in double precision using $8 \mathrm{CPU}$ cores $(2.4 \mathrm{GHz}$ Intel Ivy Bridge cores) in 1000 quasi-static time increments. The required wall-clock time ranged from 15 min for the coarsest mesh to 105 min for the finest mesh.

Figure 6 shows the predicted force versus displacement traces for the different meshes; the predictions from the FE Lagrangian method of Taylor [72] are shown for comparison. The results 


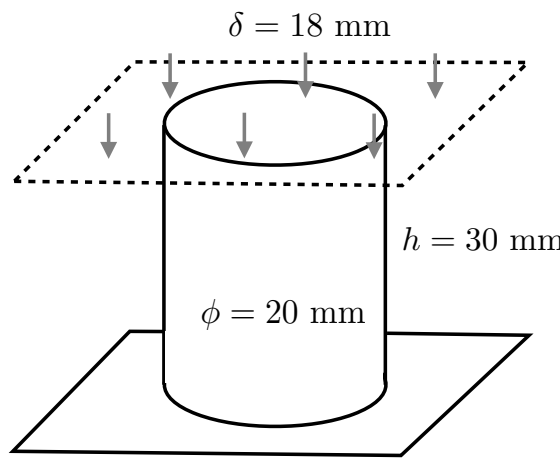

(a) Geometry

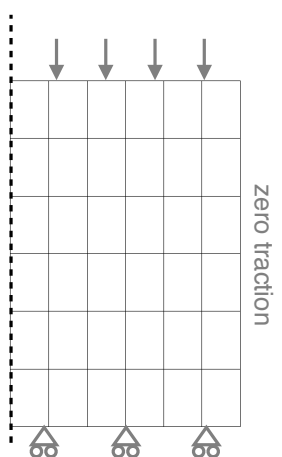

(b) 2-D axisymmetric mesh

Figure 5. Upsetting a billet: geometry, loading and mesh

\begin{tabular}{lll}
\hline Young's modulus & $E$ & $200 \mathrm{GPa}$ \\
Poisson's ratio & $\nu$ & 0.3 \\
Initial yield stress & $\sigma_{Y}$ & $700 \mathrm{MPa}$ \\
Hardening parameter & $\kappa$ & $300 \mathrm{MPa}$
\end{tabular}

Table II. Upsetting a billet: mechanical properties

can be seen to quickly converge to a mesh independent solution, close to the Taylor [72] prediction. Figures 6(b) and 6(c) show the deformed shape of the billet (mesh containing 2304 cells) for two stages of increasing deformation; the level of mesh distortion is evident, in particular, folding of the billet at the upper corner is predicted.

The predicted equivalent plastic strain distribution at $60 \%$ upset is shown in Figure 7, where for completeness both the cell-wise and vertex-wise distributions are shown; the cell-wise (average value in the cell) distribution is the direct output of the cell-centred FV method, whereas the vertexwise distributions are calculated in a post-processing interpolation step. The predictions obtained using commercial FE software Abaqus (version 6.11-1 - reduced integration bi-linear quadrilaterals) and the 9216 cell mesh are shown for comparison.

The predictions for the equivalent plastic strain distribution have been generated using the modified approach for the relative Jacobian; the predicted equivalent plastic strain distribution using the standard is almost identical; however, when the hydrostatic pressure distribution is examined, the differences between the methods can be seen. Figure 8 compares the predicted hydrostatic pressure distribution using the standard and modified approaches, where Abaqus FE results are given for reference. It is clear to see that hydrostatic pressure oscillations are present when using the standard approach, whereas the modified approach, in general, successfully eliminates the oscillations. Some minor oscillations are, however, still present.

\subsection{Crushing of a Pipe}

This test case, previously considered by Taylor [72], consists of a thick-walled pipe compressed between two parallel flat rigid frictionless platens. This case is based on the experimental study by 


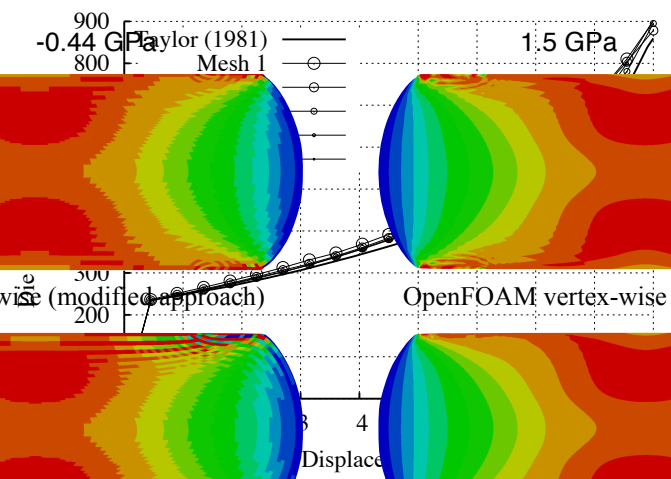

(a) Force vs. displacement

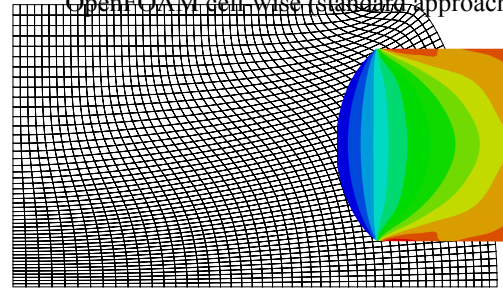

(b) Deformed geometry at $45 \%$ upset

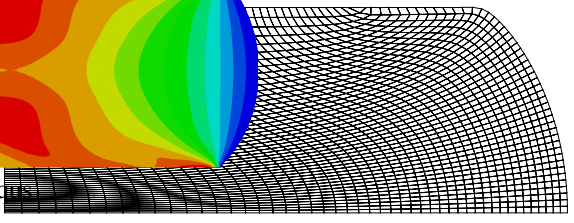

(c) Deformed geometry at $60 \%$ upset

Figure 6. Upsetting a billet: force vs. displacement, and deformed geometry at $45 \%$ and $60 \%$ upset

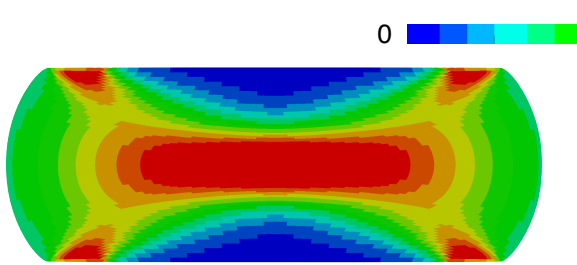

OpenFOAM cell-wise

\section{5}

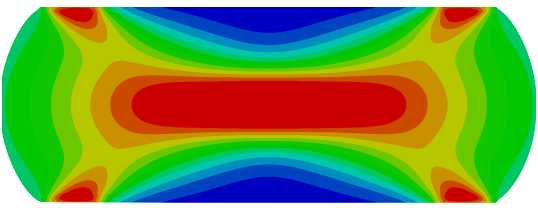

OpenFOAM vertex-wise

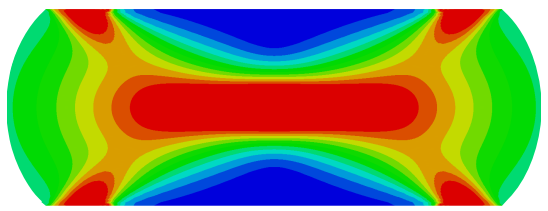

Abaqus

Figure 7. Upsetting a billet: predicted equivalent plastic strain distribution for the current OpenFOAM implementation, compared with Abaqus (FE) predictions

Peech et al. [76] and has previously been employed as a validation case for FE solvers. In the current case, the results are compared with predictions from FE commercial software Abaqus. The problem geometry is shown in Figure 9(a) and is represented here as plane strain 2-D. The inner pipe radius $r_{i}$ is $48.28 \mathrm{~mm}$ and the outer pipe radius $r_{o}$ is $57.15 \mathrm{~mm}$. The mechanical properties of the pipe are given in Table III. A rigid frictionless platen crushes the pipe through a displacement of $70 \mathrm{~mm}$ i.e. the pipe diameter is reduced from $2 \times 57.15=114.3 \mathrm{~mm}$ to $44.3 \mathrm{~mm}$ in the crushing direction. Transient effects are neglected. Due to symmetry, one quarter on the pipe cross-section is modelled 


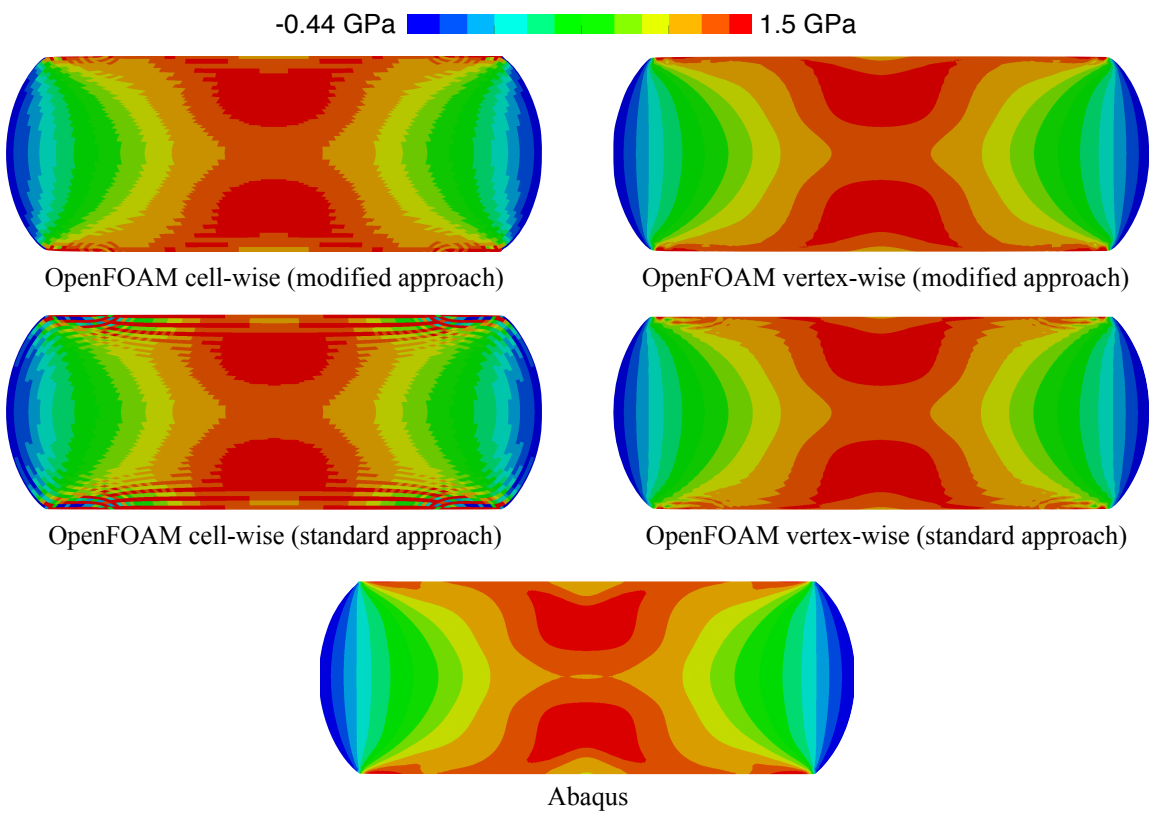

Figure 8. Upsetting a billet: predicted hydrostatic pressure distribution for the current OpenFOAM implementation, compared with Abaqus (FE) predictions

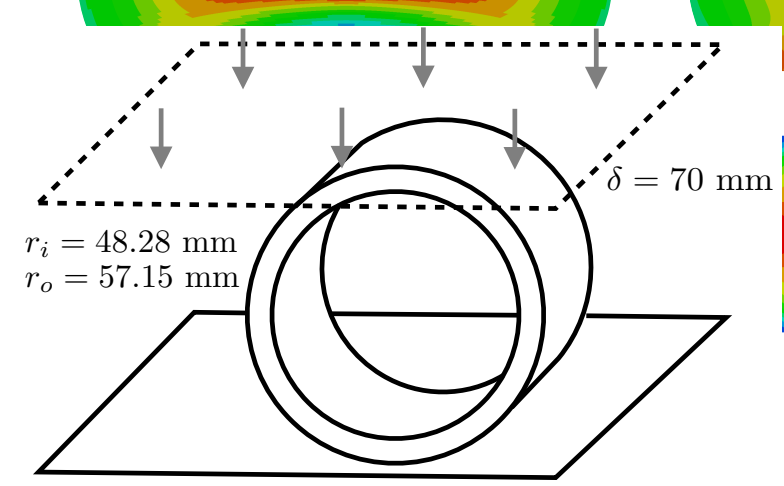

(a) Geometry

Figure 9. Crushing of a pipe: problem geometry and mesh

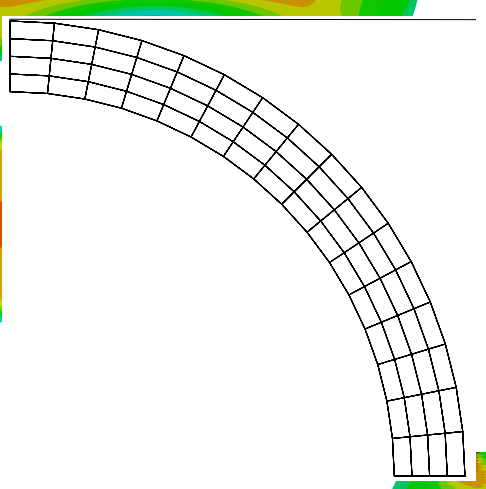

(b) 2-D Plane Strain Mesh

and symmetry plane conditions are employed. A frictionless penalty contact procedure is applied between the rigid die and the outer pipe boundary. Four separate successively refined meshes are examined, consisting of 64, 256, 1 024, and 4096 quadrilateral cells; the coarsest mesh is shown in Figure 9(b).

The models have been solved in 1000 quasi-static time increments, in double precision using 8 CPU cores (2.4 GHz Intel Ivy Bridge cores). The wall-clock time required ranged from 26 min for the coarsest mesh to 333 min for the finest mesh.

The problem has additionally been analysed using commercial FE software Abaqus and the 4096 cell mesh; the elements are assigned with reduced integration bi-linear shape functions. 


\begin{tabular}{lll}
\hline Young's modulus & $E$ & $186 \mathrm{GPa}$ \\
Poisson's ratio & $\nu$ & 0.3 \\
Initial yield stress & $\sigma_{Y}$ & $241.32 \mathrm{MPa}$ \\
Hardening & Plastic strain & Yield stress (in MPa) \\
& 0 & 241.32 \\
& 0.0035 & 275.79 \\
& 0.0083 & 301.65 \\
& 0.0133 & 318.88 \\
& 0.0182 & 344.74 \\
& 0.0281 & 361.98 \\
& 0.0380 & 379.21 \\
& 1 & 1482.37 \\
\hline
\end{tabular}

Table III. Crushing of a pipe: mechanical properties

Examining the predicted force versus displacement traces for the different meshes (Figure 10), it can be seen that there are oscillations in the force that reduce with increasing mesh density: these may be attributed to discrete faces entering the contact area; similar oscillations would be expected with standard FE contact procedures. As the mesh is refined, the predicted forces are seen to closely match the FE Abaqus fine mesh prediction.

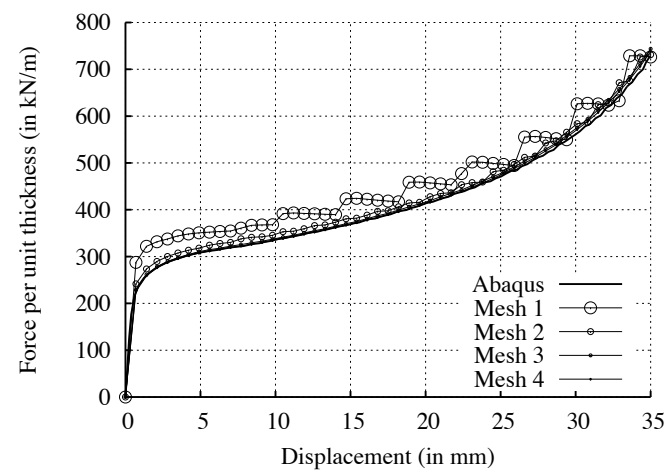

Figure 10. Crushing of a pipe: force vs. displacement

The deformed pipe geometry, at four stages of compression, is shown in Figure 11, including a comparison of the predicted hydrostatic pressure distribution with the Abaqus FE predictions. Comparing the cell-wise hydrostatic pressure distributions for the modified and standard relative Jacobian calculation methods, it can once again be seen that the standard approach suffers from erroneous oscillations, which are largely alleviated by the modified approach. The prediction using the modified approach can be seen to agree closely with the FE Abaqus predictions.

\subsection{Necking of a Cylindrical Bar}

The necking of a cylindrical bar subjected to uniaxial tension has been commonly employed as a test case to examine the ability of large strain plasticity procedures. The problem geometry is 


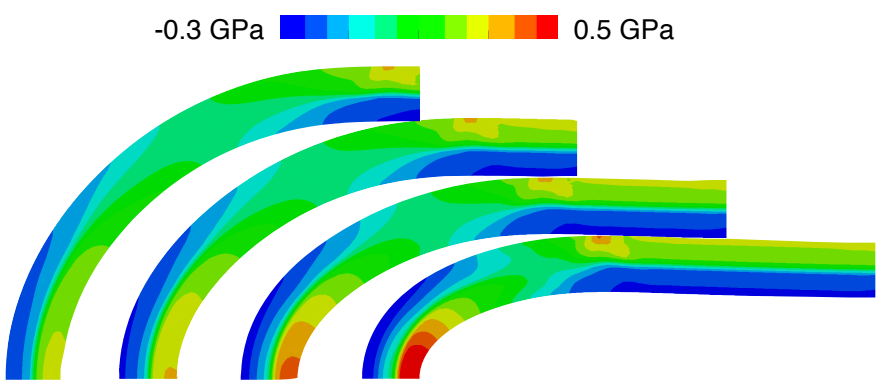

Abaqus

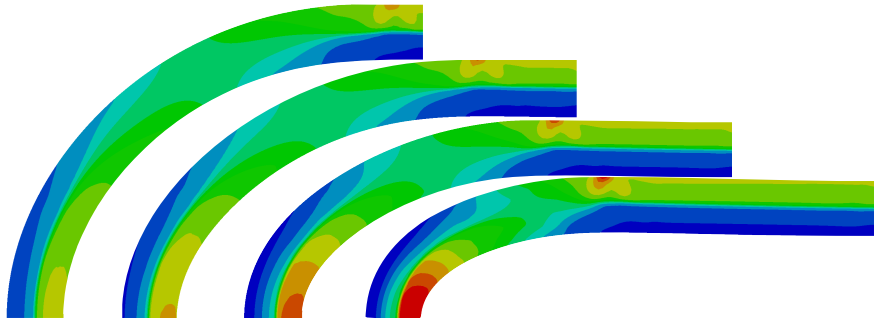

OpenFOAM vertex-wise (modified approach)

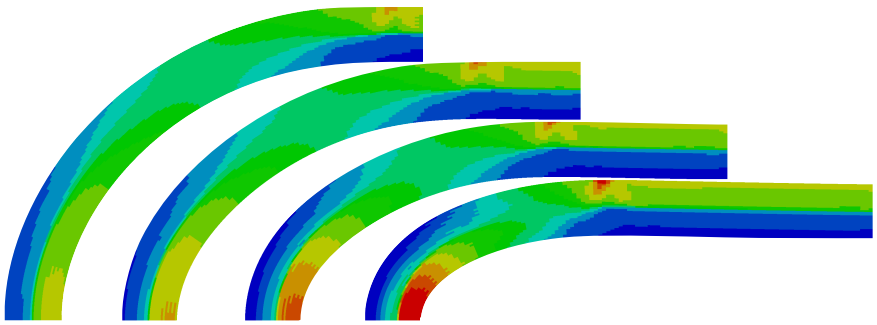

OpenFOAM cell-wise (modified approach)

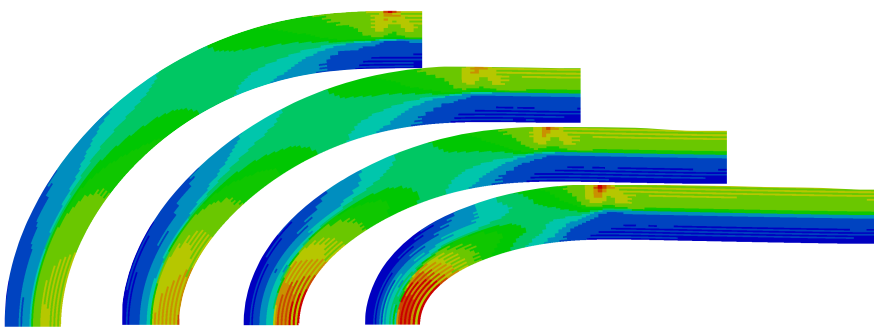

OpenFOAM cell-wise (standard approach)

Figure 11. Crushing of a pipe: deformed geometry showing hydrostatic pressure distribution

shown in Figure 12 and is represented here as axisymmetric. The initial cylindrical bar radius $r_{0}$ is $6.413 \mathrm{~mm}$ and the initial length $l_{0}$ is $53.334 \mathrm{~mm}$, where there is no initially narrowed neck region. The mechanical properties of the bar are given in Table IV. The two end surfaces of the bar are slowly displaced through a distance of $7 \mathrm{~mm}$ in opposite directions, where no tangential contraction of the ends is allowed i.e. the tangential displacement is zero. Transient effects are 
neglected. Due to symmetry, one half of the bar is simulated and symmetry plane conditions are employed. Four separate successively refined meshes are examined, consisting of 150, 600, 2400 and 9600 quadrilateral cells; the coarsest mesh is shown in Figure 12, where the mesh is refined near the expected neck, similar to the setup of Simo and Hughes [8]. The modified approach for the relative Jacobian calculation has been employed.

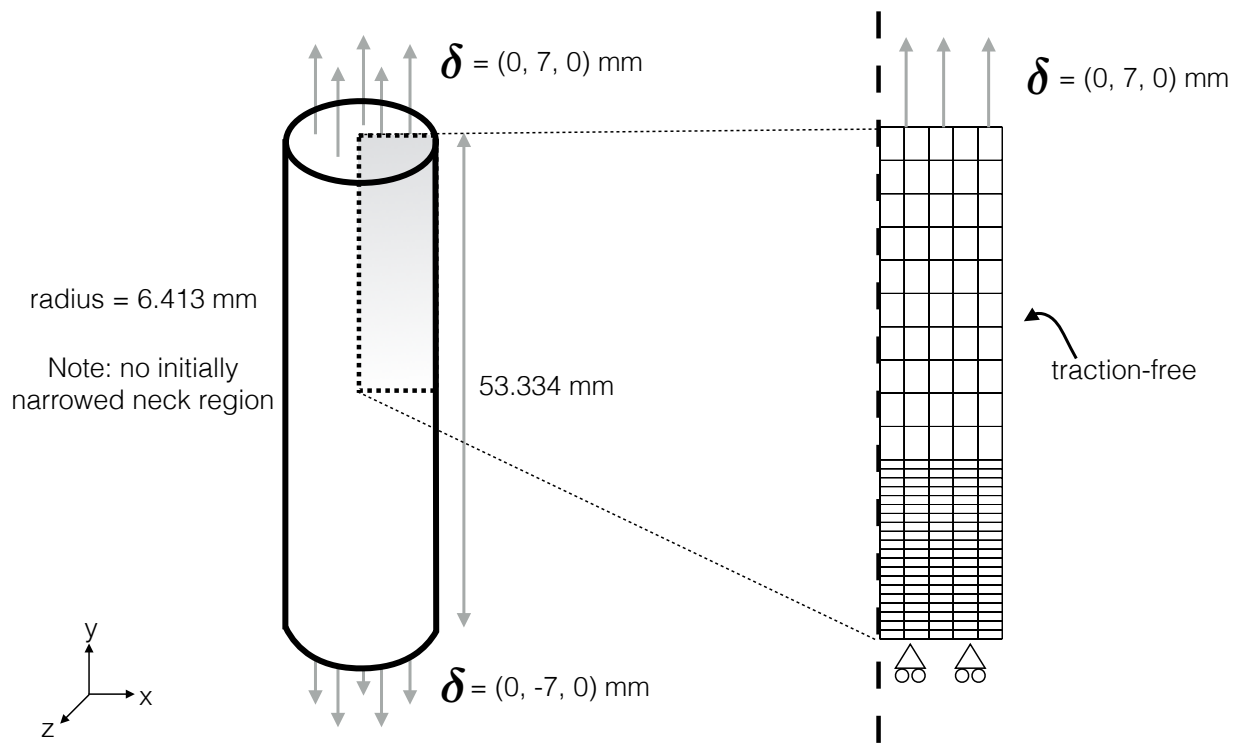

Figure 12. Necking of a cylindrical bar: problem geometry and mesh (150 cells)

\begin{tabular}{lll}
\hline Young's modulus & $E$ & $200 \mathrm{GPa}$ \\
Poisson's ratio & $\nu$ & 0.3 \\
Initial yield stress & $\sigma_{Y}$ & $451 \mathrm{MPa}$ \\
Hardening & Plastic strain & Yield stress (in MPa) \\
& 0.000 & 451 \\
& 0.006 & 476 \\
& 0.019 & 525 \\
& 0.038 & 583 \\
& 0.066 & 642 \\
& 0.147 & 710 \\
& 0.500 & 777 \\
& 1.000 & 831
\end{tabular}

Table IV. Necking of a cylindrical bar: mechanical properties

The models have been solved in 500 quasi-static time increments, in double precision using 8 $\mathrm{CPU}$ cores (2.4 GHz Intel Ivy Bridge cores). The required wall-clock time ranged from 20 min for the coarsest mesh to $5 \mathrm{~h}$ for the finest mesh.

International Journal for Numerical Methods in Engineering (0000) 
Once again for comparison, the problem has additionally been analysed using commercial FE software Abaqus and the 9600 cell mesh.

The predicted force versus axial elongation is shown for the different mesh densities in Figure 13(a); the predictions can be seen to approach the Abaqus FE fine mesh solution. In Figure 13(b), the neck radius is plotted against the axial elongation and is once again shown to match closely the Abaqus FE fine mesh solution as the mesh is refined. The predicted deformed geometry is shown in

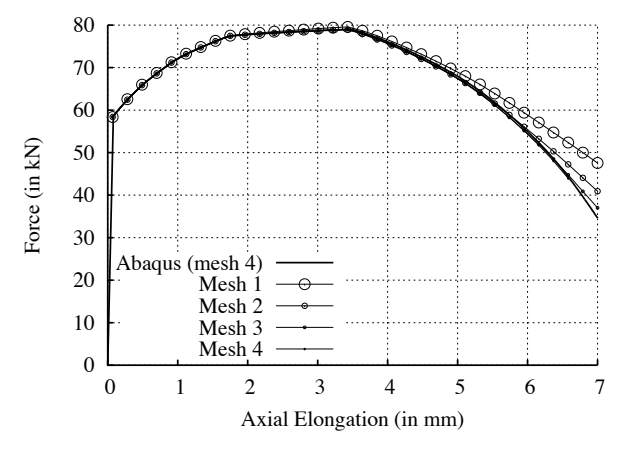

(a) Axial loading force vs. axial elongation

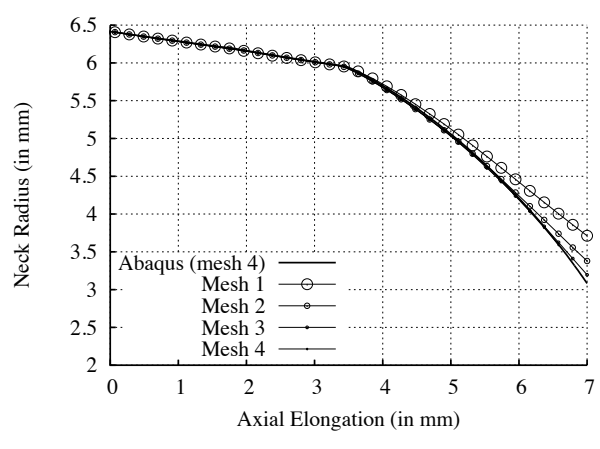

(b) Neck radius vs. axial elongation

Figure 13. Necking of a cylindrical bar: loading force and neck radius vs. axial elongation

Figure 14, where hydrostatic pressure and equivalent plastic strains distributions are compared with the Abaqus FE predictions; for completeness, the cell-wise distributions are also shown.

\subsection{Flat Rolling of Wire}

This test case comprises the flat rolling of an initially cylindrical wire, and highlights the capabilities of the method for the nontrivial 3-D problems. The problem geometry, shown in Figure 15(a), consists of a cylindrical wire, initial diameter $\phi_{w}=2.7 \mathrm{~mm}$, rolled between two cylindrical rollers, diameters $\phi_{r}=178 \mathrm{~mm}$. The gap between the rollers is $\delta=1.89 \mathrm{~mm}$ corresponding to a linear wire reduction of $30 \%$. Due to symmetry, only one quarter of the domain is simulated. Transient effects are included. The roller, represented here as a rigid surface, is rotating at $6.28 \mathrm{rad} / \mathrm{s}$, corresponding to 60 revolutions per minute. A penalty contact procedure is applied between the rigid roller and the outer wire boundary, with a Coulomb friction coefficient of 0.1. The mechanical properties of the wire are given in Table V. Five successively refined meshes are examined, consisting of 780 , $2550,19500,154$ 200, and 1230000 hexahedral cells; the second coarsest mesh is shown in Figure 15(b). In the initial stage of the simulation (over the first 40 time-steps), the roller is lowered to the required gap and accelerated to the specified angular velocity; subsequently, upstream and downstream tensions are applied to the wire. The simulation proceeds until the predicted quantities, such as force, torque and wire cross-section, have reached steady-state. The modified approach for the relative Jacobian calculation has been employed.

The predicted steady-state deformed cross-section of the wire is shown in Figure 16; the equivalent plastic strain in the wire cross-section is shown in Figure 16(a), where bands of localised plasticity, known as the blacksmith's cross, can be seen forming an $X$ pattern, e.g. see [77]; the predicted hydrostatic pressure distribution is shown in Figure 16(b); for completeness, both cellwise and vertex-wise field distributions are shown. 


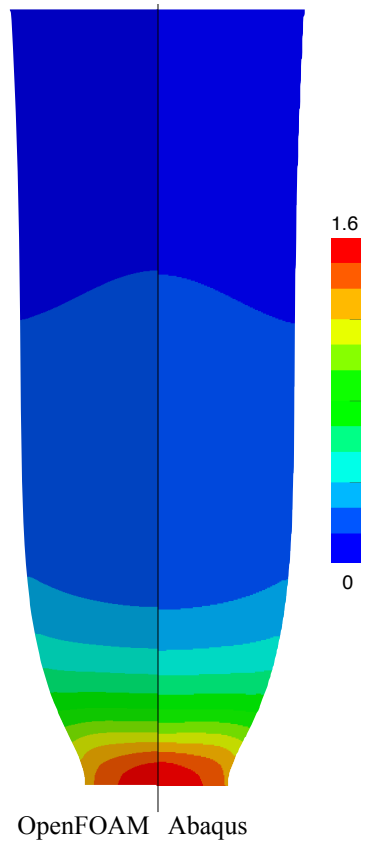

(a) Plastic strain: OpenFOAM FV (left) and Abaqus FE (right)

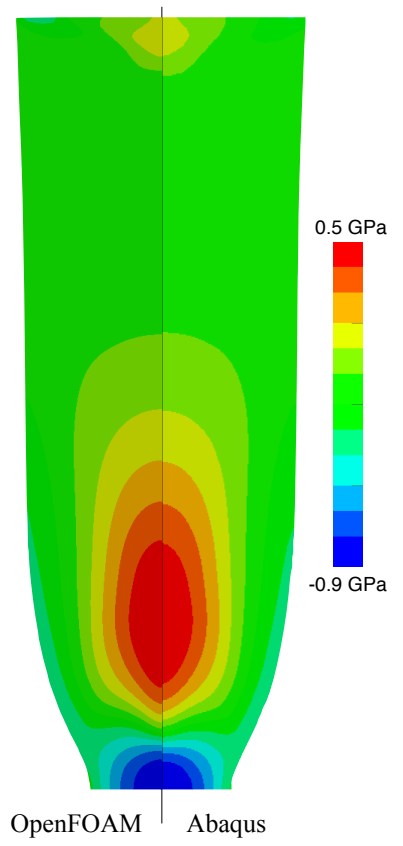

(b) Pressure: OpenFOAM FV (left) and Abaqus FE (right)

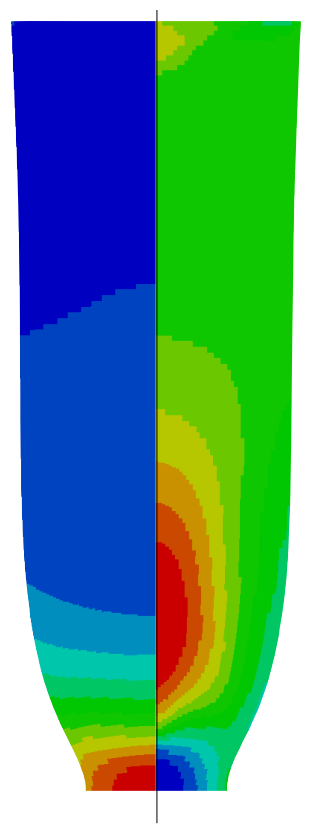

(c) Cell-wise distributions: plastic strain (left) and pressure (right)

Figure 14. Necking of a cylindrical bar: predicted deformed geometry, showing hydrostatic pressure and equivalent plastic strain distributions

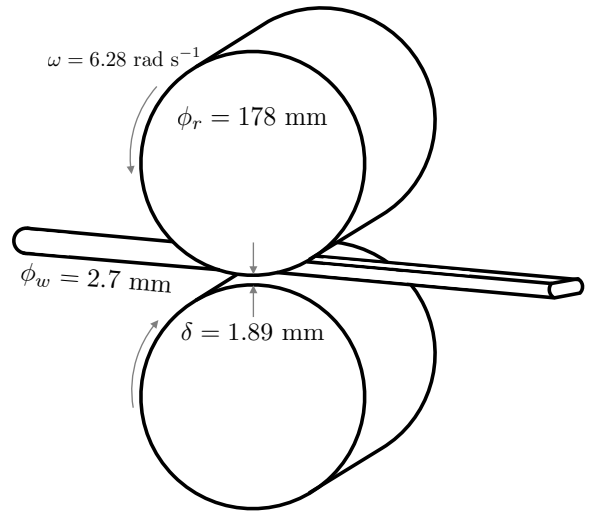

(a) Problem definition

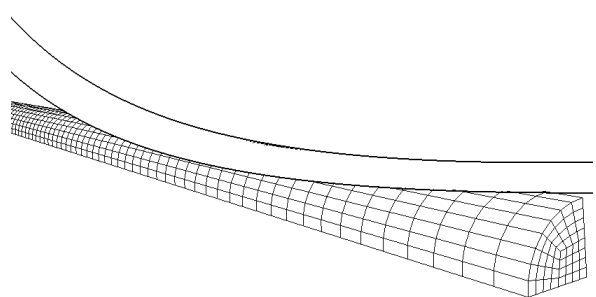

(b) Wire mesh (2 550 cells) and rigid roller

Figure 15. Flat rolling of wire: problem geometry and mesh

Using Richardson extrapolation [78] based on the solutions from the two finest meshes (mesh 4 and mesh 5), the asymptotic mesh independent predictions for the roller force, torque and power are calculated to be $16.35 \mathrm{kN}, 62.85 \mathrm{Nm}$ and $394.70 \mathrm{~W}$ respectively. Using these extrapolated predictions as a reference, the discretisation error is shown to reduce at the expected second-order rate (Figure 17). 


\begin{tabular}{lll}
\hline Young's modulus & $E$ & $210 \mathrm{GPa}$ \\
Poisson's ratio & $\nu$ & 0.3 \\
Initial yield stress & $\sigma_{Y}$ & $1.3 \mathrm{GPa}$ \\
Hardening & Plastic strain & Yield stress (in GPa) \\
& 0 & 1.3 \\
& 0.01 & 1.5 \\
& 0.1 & 1.69 \\
& 0.5 & 1.64 \\
& 0.88 & 2.11 \\
\hline
\end{tabular}

Table V. Flat rolling of wire: mechanical properties

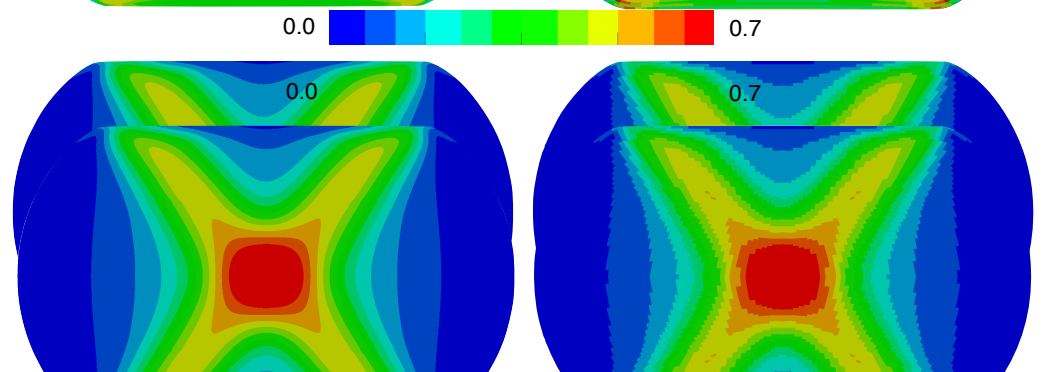

(a) Equivalent plastic strain: comparison of vertex-wise (left) and cell-size (right) distributions

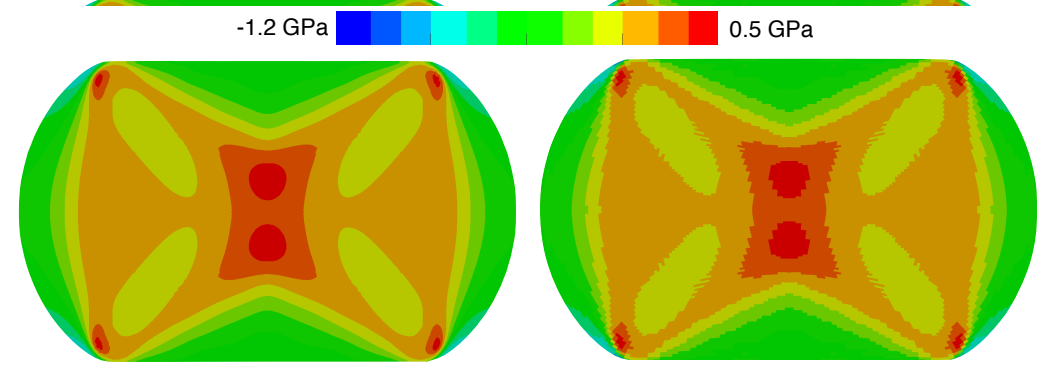

(b) Hydrostatic pressure: comparison of vertex-wise (left) and cell-size (right) distributions

Figure 16. Flat rolling of wire: predicted deformed geometry, showing equivalent plastic strain and hydrostatic pressure distributions

The steady-state contact pressure distribution between the roller and the wire is shown in Figure 18(a); a characteristic horse-shoe shaped high pressure region around the front perimeter of the contact region can be seen, consistent with previous studies, e.g. see [79]. Figure 18(b) shows the frictional shear traction vectors in the contact region. In the incoming region of the contact, the shear traction vectors are aligned with the roller surface velocity direction and have greatest magnitude. Near the exit region of the contact, the shear traction vectors flip direction, consequently, there is a region of no-slip located near the contact exit. This no-slip point can be theoretically explained by considering that the downstream wire velocity is greater than the roller surface velocity, whereas the upstream wire velocity is less than roller surface velocity; hence, at some point in the contact region, the wire surface velocity and roller surface velocity must be equal, resulting in a no-slip point. 


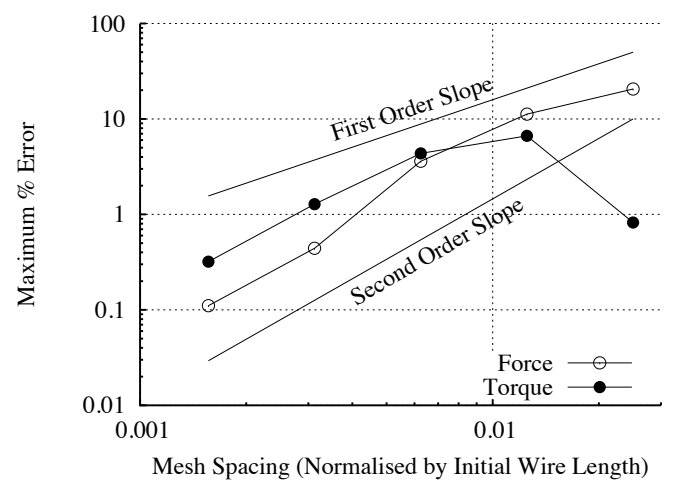

Figure 17. Flat rolling of wire: error reduction in predicted force as the mesh is refined

\subsection{Impact of cylinder against a rigid wall}

The final test consists of a cylindrical bar of copper impacting a rigid wall, where the initial radius $r_{0}=3.2 \mathrm{~mm}$, the initial length $l_{0}=32.4 \mathrm{~mm}$, and the initial velocity $v=227 \mathrm{~m} / \mathrm{s}$. The case highlights the applicability of the current method for impact scenarios. A schematic of the problem geometry is shown in Figure 19 and is represented here as axisymmetric; four successively refined quadrilateral meshes are employed, containing 90,360, 1 440, and 5760 cells. The mechanical properties are given in Table VI. Transient effects are included. A frictionless penalty contact procedure is applied between the rigid ground and the cylinder lower boundary. The modified approach for the relative Jacobian calculation has been employed.

The model has been solved in 1000 time increments, in double precision using 8 CPU core $(2.4$ $\mathrm{GHz}$ Intel Ivy Bridge cores), with wall-clock times ranging from 10 to $90 \mathrm{~min}$.

\begin{tabular}{lll}
\hline Initial density & $\rho$ & $8930 \mathrm{~kg} / \mathrm{m}^{3}$ \\
Young's modulus & $E$ & $117 \mathrm{GPa}$ \\
Poisson's ratio & $\nu$ & 0.35 \\
Initial yield stress & $\sigma_{Y}$ & $400 \mathrm{MPa}$ \\
Hardening modulus & $\kappa$ & $100 \mathrm{MPa}$
\end{tabular}

Table VI. Impact of cylinder against a rigid wall: mechanical properties

The predicted deformed geometry is shown for four separate time instances in Figure 20, where the numerical results predicted by Aguirre et al. [54] are shown for comparison. Table VII compares the final end radius of the bar at $80 \mu$ s, for the 5760 cell mesh, to numerical results from other methodologies $[54,80]$, where good agreement is found.

\section{DISCUSSION \& CONCLUSIONS}

In this paper, a Lagrangian cell-centred FV discretisation method with segregated solution methodology is presented. The governing equations are described in updated Lagrangian form 


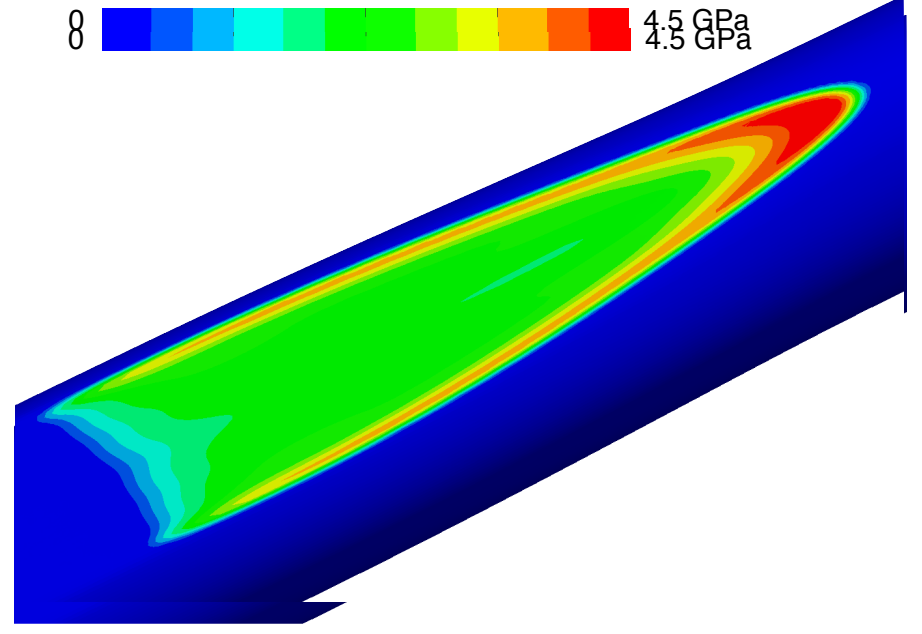

(a) Contact pressure distribution showing horse-shoe shaped high pressure region

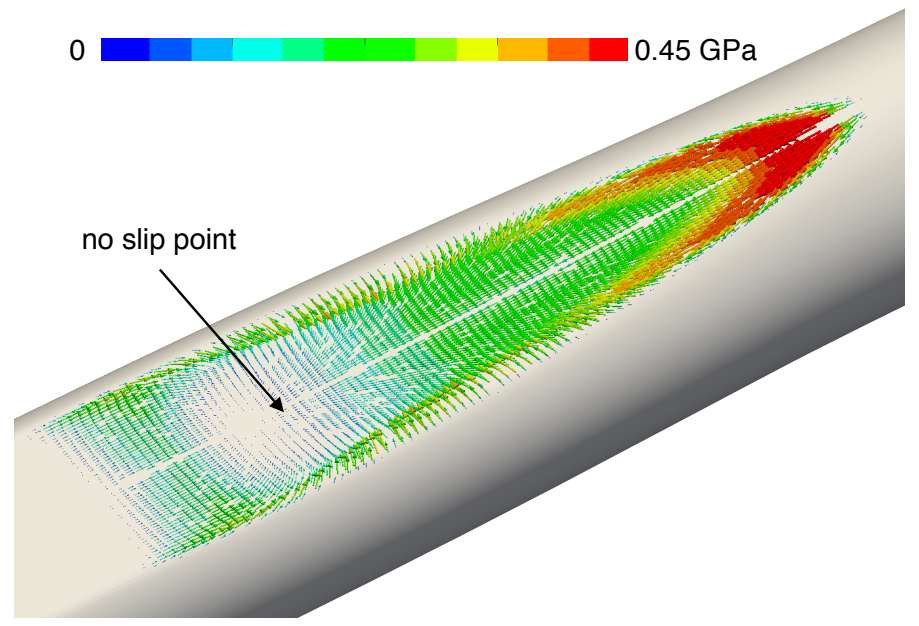

(b) Contact frictional shear traction vectors

Figure 18. Flat rolling of wire: contact pressures and shear tractions

Method

Bonet and Burton [80] FE method - tetrahedra

Bonet and Burton [80] FE method - hexahedra

Bonet and Burton [80] FE method average nodal pressure

Aguirre et al. [54] FV method

Current OpenFOAM FV method
Predicted radius (in $\mathrm{mm}$ )

5.55

6.95

6.99

6.98

7.14

Table VII. Impact of cylinder against a rigid wall: predicted end radius at $80 \mu \mathrm{s}$

and discretised in space using a cell-centred FV method. An hyperelastoplastic constitutive law is employed, with a rate-independent isotropic hyperelastic $J_{2}$ elastoplastic constitutive relation. 


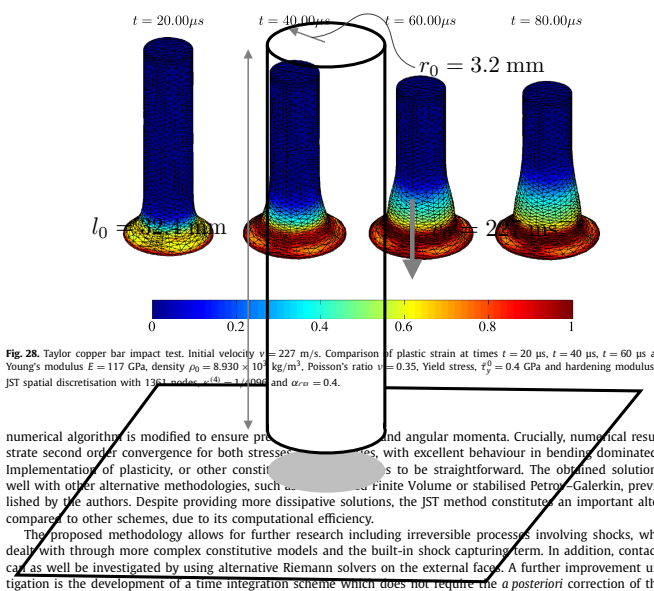

Figure 19. Impact of cylinder against a rigid wall: problem geometry and mesh (image adapted from [54])

The developed methodology has been tested on six elastoplastic benchmark test cases, where the spatial discretisation has been shown to be second-order accurate. For the cases examined, the predictions of the new method agree well with the available analytical solutions and comparative numerical solutions. A newly proposed modification to the discretisation of the relative Jacobian has been found to alleviate the issue of erroneous oscillations appearing in the predicted hydrostatic pressure distribution. From the test cases examined, the new method can be considered as a practical alternative to standard FE methods for simulation of metal forming problems.

\subsection{Limitations}

The proposed modification to the discretisation of the relative Jacobian has been found, in general, to alleviate erroneous hydrostatic pressure oscillations; however, some minor oscillations can remain. It is expected that extending the approach to use a hybrid pressure-displacement method (e.g. see $[30,56])$ would fully resolve this issue and potentially result in a more efficient procedure. Additional future improvements will focus on the inclusion of adaptive/dynamic mesh procedures to maintain the mesh quality; combining one of the many of mesh motion solvers already employed in FV procedures with a conservative mapping polyhedral method (e.g. [81]) would increase the capabilities for more severe deformation cases.

\section{ACKNOWLEDGEMENTS}

Financial support is gratefully acknowledged from Bekaert through the University Technology Centre (UTC), and the Irish Centre for Composites Research (IComp). Additionally, the authors wish to acknowledge the DJEI/DES/SFI/HEA Irish Centre for High-End Computing (ICHEC) for the provision of computational facilities and support. 


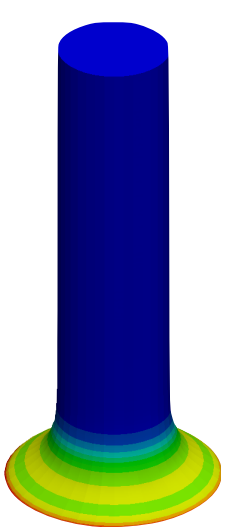

$20 \mu s$

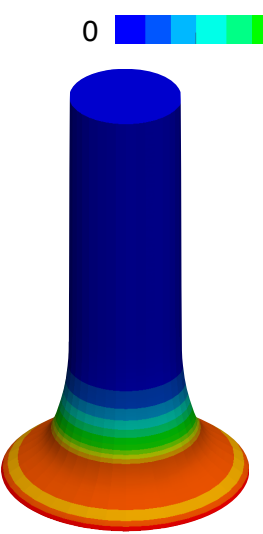

$40 \mu s$

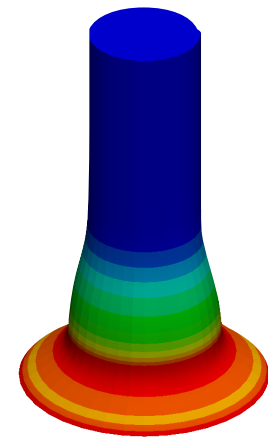

$60 \mu s$

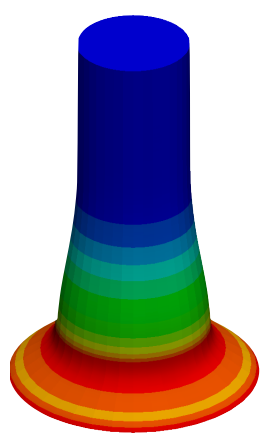

$80 \mu s$

(a) Deformed geometry predicted by the current method
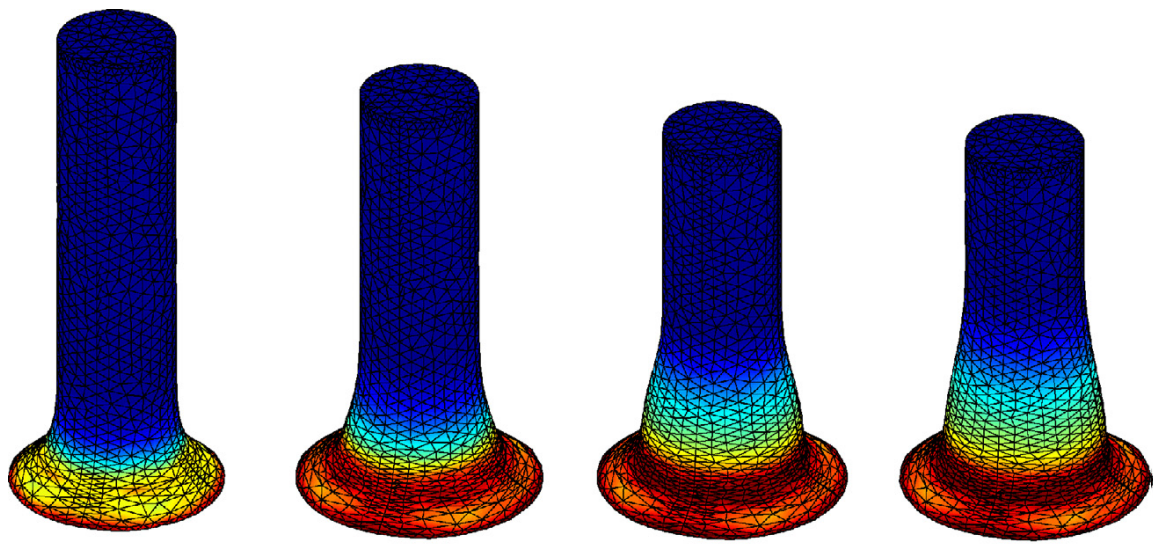

(b) Deformed geometry predicted by Aguirre et al. [54]

Figure 20. Impact of cylinder against a rigid wall: deformed geometry showing the equivalent plastic strain distribution

\section{A. APPENDIX: RECONSTRUCTION OF THE CELL-CENTRE GRADIENT OF DISPLACEMENT INCREMENT}

Within a control volume, the face-centred value of a tensor field $\phi$ may be obtained through a Taylor series expansion (higher order terms neglected) about the cell-centre:

$$
\phi_{P}+\left(\boldsymbol{r}_{f}-\boldsymbol{r}_{P}\right) \cdot(\nabla \phi)_{P}=\phi_{f}
$$

where $\boldsymbol{r}_{f}$ is the positional vector of the face-centre, and $\boldsymbol{r}_{P}$ is the positional vector of the cell-centre, as shown schematically in Figure 2; subscript $f$ indicates a face-centre value, whereas subscript $P$ indicates a cell-centre value. 
Summing over all faces in the cell and multiplying (dot product) by the term $\frac{1}{\left|\boldsymbol{\Gamma}_{f}\right|} \boldsymbol{\Gamma}_{f} \boldsymbol{\Gamma}_{f}$ results in:

$$
\left(\sum_{F} \frac{1}{\left|\boldsymbol{\Gamma}_{f}\right|} \boldsymbol{\Gamma}_{f} \boldsymbol{\Gamma}_{f}\right) \cdot \phi_{P}+\left(\sum_{F} \frac{1}{\left|\boldsymbol{\Gamma}_{f}\right|} \boldsymbol{\Gamma}_{f} \boldsymbol{\Gamma}_{f} \cdot\left(\boldsymbol{r}_{f}-\boldsymbol{r}_{P}\right)\right) \cdot[\boldsymbol{\nabla}(\phi)]_{P}=\sum_{F} \boldsymbol{\Gamma}_{f}\left(\boldsymbol{n}_{f} \cdot \boldsymbol{\phi}_{f}\right)
$$

484

where $\boldsymbol{\Gamma}_{f}$ are the face area vectors, and the face unit normals are $\boldsymbol{n}_{f}=\frac{\boldsymbol{\Gamma}_{f}}{\left|\boldsymbol{\Gamma}_{f}\right|}$.

The second term on the left-hand side of the Equation 26 is small in comparison to the first term; in fact, for a linear variation of $\phi$, the second term is zero. Neglecting this term, the relation becomes:

$$
\left(\sum_{F} \frac{1}{\left|\boldsymbol{\Gamma}_{f}\right|} \boldsymbol{\Gamma}_{f} \boldsymbol{\Gamma}_{f}\right) \cdot \phi_{P}=\sum_{F} \boldsymbol{\Gamma}_{f}\left(\boldsymbol{n}_{f} \cdot \phi_{f}\right)
$$

Multiplying both sides of Equation 27 by the inverse of the term $\left(\sum_{F} \frac{1}{\left|\boldsymbol{\Gamma}_{f}\right|} \boldsymbol{\Gamma}_{f} \boldsymbol{\Gamma}_{f}\right)$ leads to:

$$
\phi_{P}=\left(\sum_{F} \frac{1}{\left|\boldsymbol{\Gamma}_{f}\right|} \boldsymbol{\Gamma}_{f} \boldsymbol{\Gamma}_{f}\right)^{-1} \cdot \sum_{F} \boldsymbol{\Gamma}_{f}\left(\boldsymbol{n}_{f} \cdot \phi_{f}\right)
$$

During the derivation of the modified relative Jacobian discretisation, Equation 18 is constructed from Equation 28, where the cell-centred tensor $\phi_{P}$ is considered to be the reconstructed gradient of displacement increment $\widehat{\nabla(\Delta \boldsymbol{u})}$, and the face-centred normal vector $\left(\boldsymbol{n}_{f} \cdot \boldsymbol{\phi}_{f}\right)$ is considered to be the normal gradient of displacement increment at the face $[\boldsymbol{n} \cdot \boldsymbol{\nabla}(\Delta \boldsymbol{u})]_{f}$, approximated using central differencing.

\section{B. REFERENCES}

[1] S. Takagi, K. Sugiyama, S. Li, and Y. Matsumoto. A review of full Eulerian methods for fluid structure interaction problems. ASME. J. Appl. Mech., 79(1):010911, 2011. doi: 10.1115/1.4005184.

[2] O.C. Zienkiewicz. Flow formulation for numerical solution of forming processes. Numerical Analysis of Forming Processes, 1:1-44, 1984.

[3] S. Kobayashi and Taylan Altan S.O. Metal Forming and the Finite-Element Method. Oxford Series on Advanced Manufacturing. Oxford University Press, USA, 1989. ISBN 9780195364576. URL https://books.google.ie/books?id=efUEXAue 9BAC.

[4] H.J. Antunez. Linear elements for metal-forming problems within the flow approach. Computer Methods in Applied Mechanics and Engineering, 190(5-7):783 - 801, 2000. doi: http://dx.doi.org/10.1016/S0045-7825(99)00446-6. URL http://www . sciencedirect.com/science/article/pii/s0045782599004466. 
[5] K.S. Al-Athel and M.S. Gadala. Eulerian volume of solid (vos) approach in solid mechanics and metal forming. Computer methods in applied mechanics and engineering, 200:2145-2159, 2011.

[6] J.L. Chenot, M. Bernacki, P.O. Bouchard, L. Fourment, E. Hachem, and E. Perchat. Recent and future developments in finite element metal forming simulation. In $11^{\text {th }}$ International Conference on Technology of Plasticity, ICTP 2014, Nagoya Congress Center, Nagoya, Japan, 2014.

[7] P.M. Naghdi. A critical review of the state of finite plasticity. Journal of Applied Mathematics and Physics, 41(3):315-394, 1990.

[8] J. C. Simo and T. J. R. Hughes. Computational Inelasticity, volume 7. Springer-Verlag, New York, 1998.

[9] T. Belytschko, W. Kam Liu, and B. Moran. Nonlinear Finite Elements for Continua and Structures. Wiley, $2^{\text {nd }}$ edition, 2000.

[10] J. A. Trangenstein. The riemann problem for longitudinal motion in an elastic-plastic bar. Comput. Mech., 12:180-207, 1991.

[11] R. Pember and J. A. Trangenstein. Numerical algorithms for strong discontinuities in elasticplastic solids. J. Comput. Phys., 103(1):63-89, 1992.

[12] J. A. Trangenstein. Second-order godunov algorithm for two-dimensional solid mechanics. Comput. Mech., 13:343-359, 1994.

[13] P. Colella and J. A. Trangenstein. A higher-order godunov method for modeling finite deformation in elastic-plastic solids. Comm. Pure Appl. Math., 44:41-100, 1991.

[14] A.J. Williams, T.N. Croft, and M. Cross. Computational modelling of metal extrusion and forging processes. Journal of Materials Processing Technology, 125-126:573-582, 2002. doi: http://dx.doi.org/10.1016/S0924-0136(02)00401-6. URL http://www . sciencedirect.com/science/article/pii/s0924013602004016.

[15] H. Bašić, I. Demirdžić, and S. Muzaferija. Finite volume method for simulation of extrusion processes. International Journal for Numerical Methods in Engineering, 62(4):475-494, 2005. doi: 10.1002/nme.1168. URL http: / / dx. doi.org/10.1002/nme.1168.

[16] A.K. Khalifeh and J.R. Clermont. Numerical simulations of non-isothermal three-dimensional flows in an extruder by a finite-volume method. Journal of Non-Newtonian Fluid Mechanics, 126(1):7 - 22, 2005. doi: http://dx.doi.org/10.1016/j.jnnfm.2004.12.002.

[17] S. Lou, G. Zhao, R. Wang, and X. Wu. Modeling of aluminum alloy profile extrusion process using finite volume method. Journal of Materials Processing Technology, 206(13):481 - 490, 2008. doi: http://dx.doi.org/10.1016/j.jmatprotec.2007.12.084. 
[18] M.M. Martins, J.D. Bressan, S.T. Button, and A. Ivanković. Extrusion process by finite volume method using OpenFOAM software. AIP Conference Proceedings, 1315(1):14611466, 2011. doi: http://dx.doi.org/10.1063/1.3552393. URL http://scitation.aip. org/content/aip/proceeding/aipcp/10.1063/1.3552393.

[19] I. Demirdžić, D. Martinović, and A. Ivanković. Numerical simulation of thermal deformation in welded workpiece (in Croatian). Zavarivanje, 31:209-219, 1988.

[20] S.B. Beale and S.R. Elias. Stress distribution in a plate subject to uniaxial loading, phoenics. J. Comput. Fluid Dyn. Appl., 3(3):255-287, 1990.

[21] Y. D. Fryer, C. Bailey, M. Cross, and C. H. Lai. A control volume procedure for solving elastic stress-strain equations on an unstructured mesh. Applied mathematical modelling, 15(11-12): 639-645, 1991.

[22] D.B. Spalding. Simulation of fluid flow, heat transfer and solid deformation simultaneously. In NAFEMS Conference No. 4, Brighton, UK, 1993.

[23] I. Demirdžić and D. Martinović. Finite volume method for thermo-elasto-plastic stress analysis. Computer methods in applied mechanics and engineering, 109:331-349, 1993.

[24] A. Ivanković, I. Demirdžić, J. G. Williams, and P. S. Leevers. Application of the finite volume method to the analysis of dynamic fracture problems. International journal of fracture, 66(4): 357-371, 1994.

[25] J.H. Hattel and P.N. Hansen. A control volume-based finite difference method for solving the equilibrium equations in terms of displacements. Appl. Math. Model., 19:210-243., 1995.

[26] M. A. Wheel. A geometrically versatile finite volume formulation for plane elastostatic stress analysis. The Journal of Strain Analysis for Engineering Design, 31(2):111-116, 1996.

[27] A. Ivanković and G.P. Venizelos. Rapid crack propagation in plastic pipe: predicting full-scale critical pressure from s4 test results. Engineering Fracture Mechanics, 59(5):607 - 622, 1998.

[28] H. G. Weller, G. Tabor, H. Jasak, and C. Fureby. A tensorial approach to computational continuum mechanics using object orientated techniques. Computers in Physics, 12(6):620 $631,1998$.

[29] H. Jasak and H. G. Weller. Application of the finite volume method and unstructured meshes to linear elasticity. International Journal for Numerical Methods in Engineering, pages 267-287, 2000 .

[30] I. Bijelonja, I. Demirdžić, and S. Muzaferija. A finite volume method for large strain analysis of incompressible hyperelastic materials. International Journal for Numerical Methods in Engineering, 64(12):1594-1609, 2005.

[31] A. Karač and A. Ivanković. Investigating the behaviour of fluid-filled polyethylene containers under base drop impact: A combined experimental/numerical approach. International Journal of Impact Engineering, 36(4):621-631, 2009. 
[32] V. Kanyanta, A. Ivanković, and A. Karač. Validation of a fluid-structure interaction numerical model for predicting flow transients in arteries. Journal of Biomechanics, 42(11):1705-1712, 2009.

[33] A. Kelly and M. J. O’Rourke. Two system, single analysis, fluid-structure interaction modelling of the abdominal aortic aneurysms. Proceedings of the Institution of Mechanical Engineers Part H - Journal of Engineering in Medicine, 224(H8):955-970, 2010.

[34] W. Pan, M.A. Wheel, and Y. Qin. Six-node triangle finite volume method for solids with a rotational degree of freedom for incompressible material. Computers and Structures, 88(2324):1506-1511, 2010. ISSN 0045-7949. doi: http://dx.doi.org/10.1016/j.compstruc.2010.08. 001. Special Issue: Association of Computational Mechanics United Kingdom.

[35] S. Das, S. R. Mathur, and J. Y. Murthy. An unstructured finite-volume method for structureelectrostatics interactions in mems. Numerical Heat Transfer, Part B: Fundamentals: An International Journal of Computation and Methodology, 60:425-451, 2011.

[36] P. Cardiff, A. Karač, and A. Ivanković. Development of a finite volume contact solver based on the penalty method. Computational Material Science, 64:283 - 284, 2012.

[37] Ž. Tuković, A. Ivanković, and A. Karač. Finite volume stress analysis in multi-material linear elastic body. International Journal for Numerical Methods in Engineering, 2012. doi: $10.1002 / \mathrm{nme}$.

[38] M. A. A. Cavalcante and M.-J. Pindera. Generalized finite-volume theory for elastic stress analysis in solid mechanics-part I: Framework. Journal of Applied Mechanics, 79(5), 2012. doi: $10.1115 / 1.4006805$.

[39] D. Carolan, Ž. Tuković, N. Murphy, and A. Ivanković. Arbitrary crack propagation in multiphase materials using the finite volume method. Computational Materials Science, 69:153$159,2013$.

[40] P. Cardiff, A. Karač, and A. Ivanković. A large strain finite volume method for orthotropic bodies with general material orientations. Computer Methods in Applied Mechanics and Engineering, 268:318-335, 2014. doi: 10.1016/j.cma.2013.09.008.

[41] P. Cardiff, A. Karač, D. FitzPatrick, R. Flavin, and A. Ivanković. Development of a hip joint model for finite volume simulations. Journal of Biomechanical Engineering, 136:1-8, 2014. doi: $10.1115 / 1.4025776$.

[42] P. Cardiff, A. Karač, D. FitzPatrick, R. Flavin, and A. Ivanković. Development of mapped stress-field boundary conditions based on a Hill-type muscle model. International Journal for Numerical Methods in Biomedical Engineering, 2014. doi: 10.1002/cnm.

[43] J. Byrne, P. Cardiff, A. Brabazon, and M. O’Neill. Evolving parametric aircraft models for design exploration and optimisation. Neurocomput., 142:39-47, October 2014.

[44] T. Tang, O. Hededal, and P. Cardiff. On finite volume method implementation of poroelasto-plasticity soil model. International Journal for Numerical and Analytical Methods in Geomechanics, 39(13):1410-1430, 2015. 
[45] A. Safari, Z. Tukovic, P. Cardiff, M. Walter, E. Casey, and A. Ivankovic. Interfacial separation of a mature biofilm from a glass surface a combined experimental and cohesive zone modelling approach. Journal of the Mechanical Behavior of Biomedical Materials, 54:205 - 218, 2016. doi: http://dx.doi.org/10.1016/j.jmbbm.2015.09.013. URL http://www . sciencedirect.com/science/article/pii/s1751616115003495.

[46] J. M. Nordbotten. Cell-centered finite volume discretizations for deformable porous media. International journal for numerical methods in engineering, 2014. doi: 10.1002/nme.4734.

[47] P. Cardiff, Tuković, H. Jasak, and A. Ivanković. A block-coupled finite volume methodology for linear elasticity and unstructured meshes. Computers and Structures, 0:0, 2015. Under review.

[48] Ž. Tuković, A. Karač, P. Cardiff, H. Jasak, and A. Ivanković. Parallel unstructured finitevolume method for fluid-structure interaction. Computer Methods in Applied Mechanics and Engineering, 0:0, 2016. Under review.

[49] B. Šekutkovski, I. Kostić, A. Simonović, P. Cardiff, and V. Jazarević. Three-dimensional fluidstructure interaction simulation with a hybrid RANS-LES turbulence model for applications in transonic flow domain. Aerospace Science and Technology, 49:1 - 16, 2016.

[50] I. Demirdžić. On the discretization of the diffusion term in finite-volume continuum mechanics. Numerical Heat Transfer, Part B: Fundamentals: An International Journal of Computation and Methodology, 68(1):1-10, 2015.

[51] I. Demirdžić. A fourth-order finite volume method for structural analysis. Applied Mathematical Modelling, 000:1-11, 2015.

[52] G. Kluth and B. Després. Discretization of hyperelasticity on unstructured mesh with a cellcentered Lagrangian scheme. Journal of Computational Physics, 229(24):9092 - 9118, 2010. doi: http://dx.doi.org/10.1016/j.jcp.2010.08.024.

[53] C. H. Lee, Gil A. J., and Bonet J. Development of a cell centred upwind finite volume algorithm for a new conservation law formulation in structural dynamics. Comput. Struct., 118:13-38, 2013.

[54] M. Aguirre, A. J. Gil, J. Bonet, and A. A. Carreño. A vertex centred finite volume JamesonSchmidt-Turkel (JST) algorithm for a mixed conservation formulation in solid dynamics. $J$. Comput. Phys., 259:672-699, 2014.

[55] M. Aguirre, A. J. Gil, J. Bonet, and C. H. Lee. An upwind vertex centred finite volume solver for Lagrangian solid dynamics. J. Comput. Phys., 300:387-422, 2015.

[56] G. Scovazzi, B. Carnes, X. Zeng, and S. Rossi. A simple, stable, and accurate linear tetrahedral finite element for transient, nearly, and fully incompressible solid dynamics: a dynamic variational multiscale approach. International Journal for Numerical Methods in Engineering, page n/a, 2015. doi: 10.1002/nme.5138. 
[57] K. Maneeratana. Development of the finite volume method for non-linear structural applications. $\mathrm{PhD}$ thesis, Imperial College London, 2000.

[58] K. Maneeratana and A. Ivanković. Finite volume method for large deformation with linear hypoelastic materials. In In Finite Volumes for Complex Applications II, Vilsmeier $R$, Benkhaldoun F, Hanel D (eds). HERMES Science Publications, pages 459-466, 1999.

[59] K. Maneeratana and A. Ivanković. Finite volume method for structural applications involving material and geometrical non-linearities. In Proceedings of European Conference on Computational Mechanics, ECCM'99, 1999.

[60] K. Maneeratana and A. Ivanković. Finite volume method for geometrically nonlinear stress analysis applications. In $7^{\text {th }}$ Annual ACME Conference, 1999.

[61] I. Bijelonja. Finite volume method analysis of large strain elasto-plastic deformation. In The $16^{\text {th }}$ DAAAM International Symposium, Opatia, Croatia, 2005.

[62] K. J. Bathe. Finite element procedures. Prentice-Hall, New Jersey, 1996.

[63] T. Elguedj and T.J.R. Hughes. Isogeometric analysis of nearly incompressible large strain plasticity. Computer Methods in Applied Mechanics and Engineering, 268(388-416), 2014.

[64] M. Kojić and K. L. Bathe. Studies of finite element procedures: Stress solution of a closed elastic strain path with stretching and shearing using the updated Lagrangian jaumann formulation. Computers \& Structures, 26(1-2):175-179, 1987. doi: http://dx.doi.org/10.1016/ 0045-7949(87)90247-1.

[65] J.C. Simo and C. Miehe. Associative coupled thermoplasticity at finite strains: Formulation, numerical analysis and implementation. Computer Methods in Applied Mechanics and Engineering, 98(1):41-104, 1992. doi: 10.1016/0045-7825(92)90170-O.

[66] I. Demirdžić and S. Muzaferija. Numerical method for coupled fluid flow, heat transfer and stress analysis using unstructured moving meshes with cells of arbitrary topology. Computer Methods in Applied Mechanics and Engineering, 125(1-4):235-255, 1995.

[67] H. Jasak. Dynamic mesh handling in OpenFOAM. In American Institute of Aeronautics and Astronautics, pages 1-10, 2007.

[68] Nam-Sua Lee and Klaus-Jrgen Bathe. Error indicators and adaptive remeshing in large deformation finite element analysis. Finite Elements in Analysis and Design, 16(2):99 - 139, 1994.

[69] C. M. Rhie and W. L. Chow. Numerical study of the turbulent flow past an airfoil with trailing edge separation. AIAA Journal, 21:1525-1532, November 1983. doi: 10.2514/3.8284.

[70] D. A. H. Jacobs. Preconditioned conjugate gradient methods for solving systems of algebraic equations. Central Electricity Research Laboratories Report, RD/L/N193/80, 1980. 
[71] P. Cardiff. Development of the Finite Volume Method for Hip Joint Stress Analysis. PhD thesis, University College Dublin, 2012. URL https://www.researchgate.net/ publication/262772501_Development_of_the_Finite_Volume_Method_ for_Hip_Joint_Stress_Analysis.

[72] L. M. Taylor. A finite element analysis for large deformation metal forming problems involving contact and friction. The Institute for Computational Engineering and Sciences, The University of Texas at Austin, 1981. ICES Report 81-15.

[73] W. Prager and P. G. Hodge. Theory of Perfectly Plastic Solids. John Wiley and Sons, 1951.

[74] Dassault Systémes Simulia Corp. Abaqus 6.11 documentation. http://www. simulia. com/products/abaqus_fea.html, 2012.

[75] H. Lippmann. Metal Forming Plasticity: Symposium Tutzing/Germany August 28 ? September 3, 1978. Springer-Verlag Berlin Heidelberg, 1979.

[76] J. M. Peech, R. E. Roener, S. D. Porofin, East G. H., and N. A. Goldstein. Local crush rigidity of pipes and elbows. In Proc. $4^{\text {th }}$ SMIRT Conference paper F-3/8, North Holland, 1977.

[77] T.S. Cao, P.O. Bouchard, and P. Montmitonnet. Ductile damage prediction in different cold forming processes. In $33^{\text {nd }}$ Congrés Francais de Mécanique, Lyon, Ireland, 2015.

[78] P. J. Roache. Quantification of uncertainty in computational fluid dynamics. Annual review of fluid mechanics, 29:123-60, 1997.

[79] B. Carlsson. The contact pressure distribution in flat rolling of wire. Journal of Materials Processing Technology, 73(13):1 - 6, 1998. doi: http://dx.doi.org/10.1016/S0924-0136(97) 00091-5.

[80] J. Bonet and A. J. Burton. A simple average nodal pressure tetrahedral element for incompressible and nearly incompressible dynamic explicit applications. Communications in Numerical Methods in Engineering, 14(5):437-449, 1998.

[81] S. Menon and D.P. Schmidt. Conservative interpolation on unstructured polyhedral meshes: An extension of the supermesh approach to cell-centered finite-volume variables. Computer Methods in Applied Mechanics and Engineering, 200(4144):2797 - 2804, 2011. doi: http: //dx.doi.org/10.1016/j.cma.2011.04.025. 